A dimensão de Gelfand-Kirillov de certas álgebras

\author{
Lucas Galvão
}



SERVIÇO DE PÓS-GRADUAÇÃO DO ICMC-USP

Data de Depósito:

Assinatura:

\title{
A dimensão de Gelfand-Kirillov de certas álgebras
}

\author{
Lucas Galvão
}

Orientador: Prof. Dr. Daniel Levcovitz

Dissertação apresentada ao Instituto de Ciências Matemáticas e de Computação - ICMC-USP, como parte dos requisitos para obtenção do título de Mestre em Ciências - Matemática. VERSÃO REVISADA 
Ficha catalográfica elaborada pela Biblioteca Prof. Achille Bassi e Seção Técnica de Informática, ICMC/USP, com os dados fornecidos pelo(a) autor(a)

G182d $\begin{aligned} & \text { Galvão, Lucas } \\ & \text { A dimensão de Gelfand-Kirillov de certas } \\ & \text { álgebras / Lucas Galvão; orientador Daniel } \\ & \text { Levcovitz. -- São Carlos, 2014. } \\ & 50 \text { p. } \\ & \text { Dissertação (Mestrado - Programa de Pós-Graduação } \\ & \text { em Matemática) -- Instituto de Ciências Matemáticas } \\ & \text { e de Computação, Universidade de São Paulo, 2014. } \\ & \text { 1. Álgebra não comutativa. 2. Crescimento de } \\ & \text { álgebras. 3. Dimensão de Gelfand-Kirillov. I. } \\ & \text { Levcovitz, Daniel, orient. II. Título. }\end{aligned}$




\section{Agradecimentos}

Primeiramente aos meus pais, por todo o apoio e incentivo, principalmente nos momentos de maior dificuldade. Não fosse por eles, certamente eu não teria chegado até aqui.

Ao meu orientador, Prof. Daniel Levcovitz, pela sua paciência, amizade, seus ensinamentos e principalmente pela confiança depositada.

Agradeço também a todos aqueles que me apoiaram durante meu período de mestrado. Porém, faço um agradecimento especial a Renan Mezabarba, pelas discussões matemáticas (ou não) na biblioteca do ICMC, onde frequentemente incomodávamos os funcionários pela altura dos risos. Também faço agradecimentos especiais a Renan Metzker e Ivan Bolorino, amigos dos tempos de graduação na UNESP, não só pelo apoio, mas também pelas horas de descontração (compartilhadas por meio virtual).

Por fim, agradeço a CAPES pelo apoio financeiro durante um ano. 


\section{Resumo}

A dimensão de Gelfand-Kirillov mede a taxa de crescimento assintótico de álgebras. Como fornece informações importantes sobre a sua estrutura, este invariante se tornou uma das ferramentas padrão no estudo de álgebras de dimensão infinita. Neste trabalho apresentamos as propriedades básicas da dimensão de Gelfand-Kirillov de álgebras e de módulos, e também mostramos o cálculo da dimensão de Gelfand-Kirillov de algumas álgebras e módulos, sendo o exemplo mais importante o cálculo da dimensão de Gelfand-Kirillov da álgebra de Weyl $A_{n}$.

Palavras-chave: Álgebra não comutativa. Crescimento de álgebras. Dimensão de GelfandKirillov. 


\section{Abstract}

The Gelfand-Kirillov dimension measures the asymptotic rate of growth of algebras. Since it provides important structural information, this invariant has become one of the standard tools in the study of infinite dimensional algebras. In this work we present the basic properties of the Gelfand-Kirillov dimension of algebras and modules, and we also show the calculation of the Gelfand-Kirillov dimension of some algebras and modules, being the most important example the calculation of the Gelfand-Kirillov dimension of the Weyl algebra $A_{n}$.

Keywords: Noncommutative algebra. Growth of algebras. Gelfand-Kirillov dimension. 


\section{CONTEÚDO}

Introdução

1 Preliminares $\quad 5$

1.1 Crescimento de Álgebras . . . . . . . . . . . . . . . . . . . . 5

1.2 A Dimensão de Gelfand-Kirillov . . . . . . . . . . . . . . . . . . . 9

2 A álgebra de Weyl e sua dimensão de Gelfand-Kirillov (via extensões iteradas de Ore) 11

2.1 A dimensão de Gelfand-Kirillov de algumas álgebras . . . . . . . . . . . . . . 11

2.2 A álgebra de Weyl e sua dimensão de Gelfand-Kirillov . . . . . . . . . . . . . 16

2.3 A álgebra de Weyl como o anel de operadores diferenciais do anel de polinômios $\mathbb{K}\left[x_{1}, \cdots, x_{n}\right] \ldots \ldots \ldots \ldots \ldots$. . . . . . . . . . . . . . . . . . .

3 Módulos e Álgebras Filtrados e Graduados $\quad 20$

3.1 A dimensão de Gelfand-Kirillov de um módulo . . . . . . . . . . . . . . . . . 20

3.2 Álgebras filtradas e graduadas . . . . . . . . . . . . . . . . . . 25

4 Álgebras quase-comutativas $\quad 36$

4.1 Álgebras quase-comutativas . . . . . . . . . . . . . . . . 36

4.2 A dimensão de Gelfand-Kirillov de $A_{n}$-módulos $\ldots \ldots$. . . . . . . . . 47 
Bibliografia 


\section{INTRODUÇÃO}

Seja $\mathbb{K}$ um corpo, e seja $A$ uma $\mathbb{K}$-álgebra finitamente gerada. Escolha um $\mathbb{K}$-subespaço de dimensão finita $V$ (com unidade) de $A$, tal que $A$ é gerado por $V$ como álgebra sobre $\mathbb{K}$. Há uma cadeia de subespaços

$$
\mathbb{K} \subseteq V \subseteq V^{2} \subseteq \ldots \subseteq V^{n} \subseteq \ldots \subseteq \bigcup_{n=0}^{\infty} V^{n}=A
$$

com $\operatorname{dim}_{\mathbb{K}}\left(V^{n}\right)<\infty$, para cada $n \in \mathbb{N}$. O comportamento assintótico da sequência $\left(\operatorname{dim}_{\mathbb{K}}\left(V^{n}\right)\right)_{n \in \mathbb{N}}$ nos dá um invariante útil da álgebra $A$, chamado dimensão de Gelfand-Kirillov de $A$, e definido por

$$
\operatorname{GKdim}(A)=\overline{\lim } \frac{\log \operatorname{dim}_{\mathbb{K}}\left(V^{n}\right)}{\log n}
$$

No Capítulo 1, primeiramente definimos o crescimento de uma função, a fim de estudar a sequência $\left(\operatorname{dim}_{\mathbb{K}}\left(V^{n}\right)\right)_{n \in \mathbb{N}}$. Então, através da introdução de uma relação de equivalência adequada, eliminamos a dependência da escolha de um subespaço gerador $V$ de $A$, tornando possível definir o crescimento de uma álgebra. Em seguida definimos a dimensão de GelfandKirillov de uma álgebra.

No Capítulo 2 apresentamos alguns resultados básicos referentes à dimensão de GelfandKirillov, assim como alguns exemplos, sendo que o mais importante é o cálculo da dimensão de Gelfand-Kirillov das álgebras de Weyl, também definida neste capítulo. 
Então, no Capítulo 3, estendemos a dimensão de Gelfand-Kirillov para módulos. Além disso, se $V$ é um subespaço de dimensão finita de uma $\mathbb{K}$-álgebra $A$, então o conjunto formado pelos subespaços $\left\{V^{n}\right\}_{n=0}^{\infty}$ fornece uma filtração natural de $A$, e o anel graduado associado

$$
\operatorname{gr}(A) \equiv \oplus_{n=0}^{\infty} V^{n} / V^{n-1}
$$

pode ser formado. Para qualquer módulo $M_{A}$ é possível construir o módulo graduado associado $\operatorname{gr}(M)_{\operatorname{gr}(A)}$. As relações entre $M_{A}$ e $\operatorname{gr}(M)_{g r(A)}$ também são estudadas no Capítulo 3.

Por fim, no Capítulo 4 nos dedicamos ao estudo de álgebras quase comutatuvas, ou seja, álgebras $A$ tais que $\operatorname{gr}(A)$ é comutativo. Também damos uma segunda demonstração do fato de que a dimensão de Gelfand-Kirillov da $n$-ésima álgebra de Weyl é $2 n$. Por fim, provamos a desigualdade de Bernstein, a qual fornece um limitante inferior para a dimensão de Gelfand-Kirillov de um módulo sobre $A$. 


\section{CAPÍTULO 1}

\section{PRELIMINARES}

\subsection{Crescimento de Álgebras}

Neste primeiro capítulo apresentaremos algumas definições e resultados básicos que serão utilizados no decorrer do presente texto. Entretanto, omitiremos as demonstrações. Ao leitor interessado, seguem as referências.

Seja $\mathbb{K}$ um corpo. Uma $\mathbb{K}$-álgebra finitamente gerada $A$ (a qual vamos assumir que é associativa e tem elemento unitário 1) com conjunto gerador $\left\{a_{1}, \cdots, a_{m}\right\}$ possui um subespaço gerador $V$ de dimensão finita (por exemplo, o $\mathbb{K}$-espaço vetorial gerado por $a_{1}, \cdots, a_{m}$ ) no sentido de que todo elemento de $A$ é uma combinação $\mathbb{K}$-linear de monômios formados pelos elementos $a_{1}, \cdots, a_{m}$. Portanto, se $V^{0}=\mathbb{K}$, e para $n \geq 1, V^{n}$ denotar o subespaço gerado por todos os monômios em $a_{1}, \cdots, a_{m}$ de comprimento $n$, então

$$
A=\bigcup_{n=0}^{\infty} A_{n} \text {, onde } A_{n}=\mathbb{K}+V+V^{2} \cdots+V^{n} .
$$

Se $A$ possui dimensão finita, então $A=A_{n}$ para algum $n$, e a função $d_{V}=\operatorname{dim}_{\mathbb{K}}\left(A_{n}\right)$ se torna estacionária. Em geral, esta função é monótona crescente, e suas propriedades podem ser usadas para distinguir entre as várias $\mathbb{K}$-álgebras. Entretanto, a função $d_{V}$ é muito específica, pois depende da escolha do subespaço gerador $V$. A dependência pode ser 
removida ao introduzirmos uma relação de equivalência adequada.

Definição 1.1.1. Seja $\Phi$ o conjunto de todas as funções $f: \mathbb{N} \rightarrow \mathbb{R}$ que são monótonas crescentes a partir de um determinado ponto e a valores positivos, ou seja, para as quais existe $n_{0}=n_{0}(f) \in \mathbb{N}$, tal que

$$
f(n) \in \mathbb{R}^{+} \quad \text { e } f(n+1) \geq f(n) \quad \text { para todo } n \geq n_{0} .
$$

Para $f, g \in \Phi$ dizemos que $f \leq^{*} g$ se, e somente se, existem $c, m \in \mathbb{N}$ tais que

$$
f(n) \leq c g(m n) \quad \text { para quase todo } n \in \mathbb{N} \text {, }
$$

e $f \sim g$ se, e somente se, $f \leq^{*} g$ e $g \leq^{*} f$. Para $f \in \Phi$ a classe de equivalência $\mathcal{G}(f) \in \Phi / \sim$ é chamada crescimento de $f$. A ordem parcial no conjunto $\Phi / \sim$ induzida por $\leq^{*} e ́$ denotada por $\leq$.

\section{Observações:}

(a) Se $f$ e $g$ são funções polinomiais, pode ser mostrado que $f$ e $g$ possuem o mesmo crescimento se, e somente se, $\operatorname{deg}(f)=\operatorname{deg}(g)$. Para um número $\gamma \geq 0$ o crescimento da função $p_{\gamma}: n \rightarrow n^{\gamma}$ é denotado por $\mathcal{P}_{\gamma}$.

(b) Para um número real positivo $\epsilon$, o crescimento de $q_{\epsilon}: n \rightarrow e^{n^{\epsilon}}$ é denotado por $\mathcal{E}_{\epsilon}$. Claramente, $\epsilon<\eta$ se, e somente se, $\mathcal{E}_{\epsilon}<\mathcal{E}_{\eta}$.

(c) Seja $f(n)=\log (n)$. Então $f \in \Phi$ e $\mathcal{G}(f)>\mathcal{P}_{0}$. É fácil ver que $\mathcal{G}(f)<\mathcal{P}_{\epsilon}$ para qualquer $\epsilon>0$.

(d) Notemos que $\mathcal{G}(f)$ e $\mathcal{G}(g)$ não precisam ser comparáveis para duas funções $f$ e $g$ em $\Phi$.

Lema 1.1.2. ([4], página 6) Seja A uma $\mathbb{K}$-álgebra finitamente gerada com subespaços geradores $V$ e $W$. Se $d_{V}(n)$ e $d_{W}(n)$ denotam as dimensões de $\sum_{i=0}^{n} V^{i}$ e $\sum_{i=0}^{n} W^{i}$, respectivamente, então $\mathcal{G}\left(d_{V}\right)=\mathcal{G}\left(d_{W}\right)$.

O fato de que o crescimento da função dimensão de uma $\mathbb{K}$-álgebra finitamente gerada independe do subespaço gerador de dimensão finita $\mathrm{V}$ considerado motiva a próxima definição. 
Definição 1.1.3. Seja A uma $\mathbb{K}$-álgebra finitamente gerada, e seja $V$ um subespaço gerador de dimensão finita de $A$. Então $\mathcal{G}(A):=\mathcal{G}\left(d_{V}\right)$ é chamado de crescimento de A. Além disso dizemos que A possui

- crescimento polinomial se $\mathcal{G}(A)=\mathcal{P}_{m}$, para algum $m \in \mathbb{N}$,

- crescimento exponencial se $\mathcal{G}(A)=\mathcal{E}_{1}$,

Exemplo 1.1.4. Seja $A=\mathbb{K}\langle x, y\rangle$ a álgebra livre em dois geradores. Então $\mathbb{K} x+\mathbb{K} y$ é um subespaço gerador de $A, e$

$$
d_{V}(n)=\operatorname{dim}_{\mathbb{K}}\left(\sum_{i=0}^{n} V^{i}\right)=1+2+2^{2}+\cdots+2^{n}=2^{n+1}-1 .
$$

$\operatorname{Logo} \mathcal{G}(A)=\mathcal{E}_{1}$

O resultado seguinte, devido a Borho e Kraft, mostra que o crescimento exponencial é o maior crescimento possível para uma álgebra finitamente gerada.

Proposição 1.1.5. Se A é finitamente gerada mas não possui dimensão finita, então $\mathcal{P}_{1} \leq \mathcal{G}(A) \leq \mathcal{E}_{1}$

Demonstração: Seja $V$ um subespaço gerador de dimensão finita para $A$, e assuma que $1 \in V$. Então

$$
d_{V}(n)=\operatorname{dim}\left(V^{n}\right) \leq \operatorname{dim}(V \otimes \cdots \otimes V)=(\operatorname{dim} V)^{n},
$$

portanto $\mathcal{G}(A)=\mathcal{G}\left(d_{V}\right) \leq \mathcal{E}_{1}$

Por outro lado, as inclusões na sequência $V^{0} \subseteq V^{1} \subseteq V^{2} \cdots$ são todas próprias, pois se $V^{n}=V^{n+1}$ para algum $n$, então $V^{n}=A$, e $A$ possui dimensão finita. Logo $d_{V}(n) \geq n$, para todo $n$, e consequentemente $\mathcal{G}(A)=\mathcal{G}\left(d_{V}\right) \geq \mathcal{P}_{1}$.

Muitas das álgebras que estudaremos terão crescimento polinomial. De modo a determinar os respectivos polinômios, o seguinte lema será útil.

Lema 1.1.6. ([4], página 7) Seja $\mathbb{Q}$ o corpo dos números racionais. 
(a) Se $0 \neq f \in \mathbb{Q}[x]$ é um polinômio de grau d, então existem números racionais $a_{0}, a_{1}, \cdots, a_{d}$ tais que

$$
f(n)=a_{d}\left(\begin{array}{l}
n \\
d
\end{array}\right)+a_{d-1}\left(\begin{array}{c}
n \\
d-1
\end{array}\right)+\cdots+a_{1}\left(\begin{array}{l}
n \\
1
\end{array}\right)+a_{0}
$$

para todo número natural $n$.

(b) As seguintes propriedades de uma função $f: \mathbb{N} \rightarrow \mathbb{Q}$ são equivalentes.

i. Existem $a_{0}, a_{1}, \cdots, a_{d} \in \mathbb{Q}$ e um inteiro $m \geq 0$ tal que para todo $n \geq m$

$$
f(n)=a_{d}\left(\begin{array}{l}
n \\
d
\end{array}\right)+a_{d-1}\left(\begin{array}{c}
n \\
d-1
\end{array}\right)+\cdots+a_{1}\left(\begin{array}{l}
n \\
1
\end{array}\right)+a_{0}
$$

ii. Existem $a_{1}, \ldots, a_{d} \in \mathbb{Q}$ e um inteiro $m \geq 0$ tal que para todo $n \geq m$

$$
f(n+1)-f(n)=a_{d}\left(\begin{array}{c}
n \\
d-1
\end{array}\right)+\cdots+a_{2}\left(\begin{array}{l}
n \\
1
\end{array}\right)+a_{1}
$$

(c) Se $f(n)$ está no formato descrito em (a) e se $f(n) \in \mathbb{Z}$ para todo $n$ suficientemente grande, então $a_{i} \in \mathbb{Z}$ para $i=0,1, \cdots, d$.

(d) Se $f(n)$ está no formato descrito em (a) e se $f(n) \in \mathbb{N}$ e $f(n+1)-f(n) \geq 0$ para todo $n$ suficientemente grande, então $a_{d}$ é um inteiro positivo.

Exemplo 1.1.7. Considere $A=\mathbb{K}\left[x_{1}, \ldots, x_{d}\right]$, a álgebra polinomial comutativa. $O$ espaço vetorial $V=\mathbb{K} x_{1}+\ldots \mathbb{K} x_{d}$ é um subespaço gerador de $A$, e é fácil ver que

$$
\operatorname{dim}\left(V^{n+1}\right)=\left(\begin{array}{c}
n+1+d-1 \\
d-1
\end{array}\right)=\left(\begin{array}{l}
n+d \\
d-1
\end{array}\right)
$$

que é um polinômio em $n$ de grau $d-1$. Como $\operatorname{dim}\left(V^{n+1}\right)=d_{V}(n+1)-d_{V}(n)$, segue da parte (b) do lema anterior que $d_{V}(n)$ é um polinômio em $n$ de grau $d ; \operatorname{logo} \mathcal{G}(A)=\mathcal{P}_{d}$. De fato, as partes (c) e (d) do lema anterior mostram que os coeficientes de $d_{V}$ são inteiros, e que seu coeficiente dominante é um inteiro positivo. 


\subsection{A Dimensão de Gelfand-Kirillov}

O crescimento de uma álgebra frequentemente é difícil de determinar e se mostra uma ferramenta de difícil aplicação quando são consideradas álgebras relacionadas, como subálgebras, quocientes, extensões de Ore, etc. Então é apropriado utilizar o comportamento assintótico das funções monótonas crescentes a fim de fazermos identificações que vão além do crescimento. Teoricamente há várias possibilidades para prosseguir, mas na prática o seguinte limite superior

$$
\overline{\lim } \frac{\log f(n)}{\log n}
$$

se mostra um dos mais úteis. No que se segue, utilizaremos a notação $\log _{n} f(n)$ para denotar $\log f(n) / \log n$.

Lema 1.2.1. ([4], página 13) Sejam $f, g \in \Phi$. Então

- $\overline{\lim } \log _{n} f(n)=\left\{\inf \rho \in \mathbb{R}, f(n) \leq n^{\rho}\right.$ para quase todo $\left.n\right\}=\left\{\inf \rho \in \mathbb{R}, \mathcal{G} \leq \mathcal{P}_{\rho}\right\}$

- $S e \mathcal{G}(f)=\mathcal{G}(g)$, então $\overline{\lim } \log _{n} f(n)=\overline{\lim } \log _{n} g(n)$

Podemos agora dar a próxima definição. Note que nela não supomos que $A$ é uma $\mathbb{K}$-álgebra finitamente gerada.

Definição 1.2.2. A dimensão de Gelfand-Kirillov de uma $\mathbb{K}$-álgebra $A$ é

$$
G K \operatorname{dim}(A)=\sup _{V} \overline{l i m} \log _{n} d_{V}(n),
$$

onde o supremo é tomado sobre todos os subespaços finitamente dimensionais $V$ de $A$.

Observação 1.2.3. O Lema 1.1.2 diz que para uma $\mathbb{K}$-álgebra finitamente gerada $B$ com subespaço gerador de dimensão finita $V$, o crescimento de $B$ independe da escolha particular de $V$. Logo

$$
\operatorname{GKdim}(B)=\overline{\lim } \log _{n} d_{V}(n)
$$


neste caso. Como todo subespaço de dimensão finita de uma $\mathbb{K}$-álgebra geral $A$, não necessariamente finitamente gerada, pode ser visto como o subespaço gerador para uma álgebra finitamente gerada $B$ de $A$, a definição da dimensão de Gelfand-Kirillov de $A$ pode ser reescrita como

$$
G K \operatorname{dim}(A)=\sup _{B}\{G K \operatorname{dim}(B), B \subset A, B \text { subálgebra finitamente gerada. }\}
$$

A dimensão de Gelfand-Kirillov de uma álgebra também pode ser infinita, como veremos no exemplo a seguir.

Exemplo 1.2.4. Seja A a álgebra do Exemplo 1.1.4, a qual é finitamente gerada. Como $d_{V}(n)=2^{n+1}-1$, temos que

$$
\overline{\lim } \log _{n} d_{V}(n)=\overline{\lim } \log _{n} 2^{n+1}-1=\overline{\lim }\left((n+1) \log _{n} 2-\log _{n} 1\right)=\infty .
$$

Logo a dimensão de Gelfand-Kirillov da álgebra acima é infinita.

No entanto, enfatizamos que nesta dissertação estamos interessados somente em $\mathbb{K}$-álgebras $A$ que são finitamente geradas.

A seguinte questão segue naturalmente: quais números reais podem ser assumidos pela dimensão de Gelfand-Kirillov de uma $\mathbb{K}$-álgebra? É fácil ver que $G K \operatorname{dim}(A)=0$ se, e somente se, $A$ é localmente de dimensão finita, ou seja, se toda subálgebra finitamente gerada possui dimensão finita. Segue da Proposição 1.1 .5 que $G K \operatorname{dim}(A) \geq 1$ para qualquer álgebra $A$ que não é localmente de dimensão finita. Além disso, temos do Exemplo 1.1.7 que todo número natural ocorre como dimensão de Gelfand-Kirillov de uma álgebra polinomial comutativa. Há dois resultados que respondem a essa pergunta para os outros números positivos.

Teorema 1.2.5. ([4], página 18) Nenhuma álgebra possui dimensão de Gelfand-Kirillov estritamente entre 1 e 2.

Teorema 1.2.6. ([4], página 21) Para qualquer número real $r \geq 2$ existe uma álgebra $A=\mathbb{K}\langle x, y\rangle /\{Z\}$ com dois geradores tal que $G K \operatorname{dim}(A)=r$. 


\section{CAPÍTULO 2}

\section{A ÁLGEBRA DE WEYL E SUA}

DIMENSÃO DE

\section{GELFAND-KIRILLOV (VIA \\ EXTENSÕES ITERADAS DE ORE)}

\subsection{A dimensão de Gelfand-Kirillov de algumas álgebras}

Para que a dimensão de Gelfand-Kirillov seja um método útil para o estudo de álgebras, é necessário conhecer seu comportamento quando passamos de uma álgebra $A$ para anéis relacionados como subálgebras, álgebras quocientes, somas diretas e extensões de Ore. Neste capítulo estas álgebras serão estudadas. Como uma aplicação obteremos o resultado fundamental deste capítulo, que é a dimensão de Gelfand-Kirillov das álgebras de Weyl.

Lema 2.1.1. Se B é uma subálgebra ou uma imagem homomorfa de uma $\mathbb{K}$-álgebra $A$, então $\operatorname{GKdim}(B) \leq G \operatorname{Kdim}(A)$. 
Demonstração: Para subálgebras a afirmação decorre como consequência imediata da definição da dimensão de Gelfand-Kirillov. Para uma álgebra quociente $\bar{A}$ de $A$, observe que qualquer conjunto de representantes das classes laterais para os elementos da base de um subespaço $\bar{V}$ de $\bar{A}$ com dimensão finita forma uma base de um subespaço de dimensão finita $V$ de $A$ que satisfaz $\operatorname{dim}\left(\bar{V}^{n}\right) \leq \operatorname{dim}\left(V^{n}\right)$, para todo $n$ natural. Então

$$
\overline{\lim } \log _{n} \operatorname{dim}\left(\bar{V}^{n}\right) \leq \overline{\lim } \log _{n} \operatorname{dim}\left(V^{n}\right)
$$

e portanto,

$$
\sup _{\bar{V}} \overline{\lim } \log _{n} \operatorname{dim}\left(\bar{V}^{n}\right) \leq \sup _{V} \overline{\lim } \log _{n} \operatorname{dim}\left(V^{n}\right)
$$

Proposição 2.1.2. Se $A_{1}$ e $A_{2}$ são $\mathbb{K}$-álgebras, então

$$
\operatorname{GKdim}\left(A_{1} \oplus A_{2}\right)=\max \left\{G K \operatorname{dim}\left(A_{1}\right), G K \operatorname{dim}\left(A_{2}\right)\right\} .
$$

Demonstração: Pelo Lema 2.1.1, temos

$$
\gamma:=\max \left\{\operatorname{GKdim}\left(A_{1}\right), G K \operatorname{dim}\left(A_{2}\right)\right\} \leq G K \operatorname{dim}\left(A_{1} \oplus A_{2}\right),
$$

e a igualdade vale se $\gamma=\infty$. Vamos assumir que $\gamma$ é finito, e seja $W$ um subespaço de dimensão finita de $A_{1} \oplus A_{2}$. Sejam $U$ e $V$ as projeções canônicas de $W$ sobre $A_{1}$ e $A_{2}$ respectivamente. Então

$$
W \subseteq U \oplus V \text { e } W^{n} \subseteq(U \oplus V)^{n}=U^{n} \oplus V^{n}
$$

Dado $\epsilon>0$, segue do Lema 1.2.1 que

$$
d_{U}(n)<n^{\gamma+\frac{\epsilon}{2}} \quad \text { e } \quad d_{V}(n)<n^{\gamma+\frac{\epsilon}{2}} .
$$

para quase todo n, pois $\overline{\lim } \log _{n} d_{U}(n) \leq G K \operatorname{dim}\left(A_{1}\right) \leq \gamma$ e o mesmo vale para para $d_{V}(n)$. Como $n^{\frac{\epsilon}{2}}>2$ para $n$ suficientemente grande,

$$
d_{W}(n) \leq d_{U}(n)+d_{V}(n) \leq 2 n^{\gamma+\frac{\epsilon}{2}}<n^{\frac{\epsilon}{2}} n^{\gamma+\frac{\epsilon}{2}}=n^{\gamma+\epsilon}
$$


para quase todo $n$. Logo $\overline{l i m} \log _{n} d_{W}(n) \leq \gamma$, pelo Lema 1.2.1. Portanto,

$$
\operatorname{GKdim}\left(A_{1} \oplus A_{2}\right)=\sup _{W} \overline{\lim } \log _{n} d_{W}(n) \leq \gamma
$$

Muitas álgebras são extensões de Ore de outras álgebras, sendo as álgebras de Weyl talvez o exemplo mais conhecido.

Definição 2.1.3. Uma $\mathbb{K}$-derivação $\delta$ de uma $\mathbb{K}$-álgebra $A$ é um $\mathbb{K}$-endomorfismo de $A$ que satisfaz

$$
\delta(a b)=\delta(a) b+a \delta(b) \text { para todo } a, b \in A .
$$

Definição 2.1.4. Seja $A$ uma $\mathbb{K}$-álgebra com uma $\mathbb{K}$-derivação $\delta . A \mathbb{K}$-álgebra $B=A[x ; \delta]$ dos polinômios $a_{0}+a_{1} x+\ldots+a_{n} x^{n}, a_{i} \in A$, sujeita à relação $x a-a x=\delta(a)$ é chamada extensão de Ore de $A$ com respeito à $\delta$.

Lema 2.1.5. Seja $A$ uma $\mathbb{K}$-álgebra com $\mathbb{K}$-derivação $\delta$, e seja $B=A[x ; \delta]$. Então $G \operatorname{Kdim}(B) \geq G \operatorname{Gdim}(A)+1$.

Demonstração: Seja $A_{f}$ uma subálgebra finitamente gerada de $A$ e seja $V$ um subespaço gerador de dimensão finita para $A_{f}$, com unidade. O subespaço de dimensão finita $W=V+\mathbb{K} x$ de $B$ satisfaz

$$
V^{n} \oplus V^{n} x \oplus \cdots \oplus V^{n} x^{n} \subseteq(V+\mathbb{K} x)^{2 n}=W^{2 n} ;
$$

$\log \mathrm{o}(n+1) d_{V}(n) \leq d_{W}(2 n)$. Então

$$
\begin{aligned}
G K \operatorname{dim}(B) & \geq \overline{\lim } \log _{n} d_{W}(n) \geq \overline{\lim } \log _{n}(n+1) d_{V}(n) \\
& =\lim _{n \rightarrow \infty} \log _{n}(n+1)+\overline{\lim } \log _{n} d_{V}(n)=1+\operatorname{GKdim}\left(A_{f}\right)
\end{aligned}
$$

e portanto,

$$
\operatorname{GKdim}(B) \geq \sup _{A_{f}}\left\{1+G K \operatorname{dim}\left(A_{f}\right)\right\}=1+\sup _{A_{f}}\left\{G K \operatorname{dim}\left(A_{f}\right)\right\}=1+G K \operatorname{dim}(A) .
$$


É natural querer saber quando a desigualdade no lema anterior se torna uma igualdade. O resultado seguinte mostra que a igualdade vale para uma grande classe de álgebras consideradas nas aplicações, em particular, para o caso $\delta=0$, isto é, para a álgebra polinomial $B=A[x]$, e para o caso quando $A$ é uma álgebra finitamente gerada.

Proposição 2.1.6. Seja A uma $\mathbb{K}$-álgebra com $\mathbb{K}$-derivação $\delta$ tal que todo subespaço de dimensão finita está contido em uma subálgebra $\delta$-estável de A. Então

$\operatorname{GKdim}(A[x ; \delta])=G \operatorname{Kdim}(A)+1$.

Demonstração: Seja $B^{\prime}$ uma subálgebra finitamente gerada de $B=A[x ; \delta]$. $\mathrm{O}$ espaço vetorial gerado pelos coeficientes dos finitos polinômios que geram $B^{\prime}$ é de dimensão finita e portanto está contido em uma subálgebra $A^{\prime} \delta$-estável finitamente gerada de $A$. Como $B^{\prime} \subseteq A^{\prime}[x ; \delta]$, o resultado segue se mostrarmos que $G K \operatorname{dim}\left(B^{\prime}\right) \leq G K \operatorname{dim}\left(A^{\prime}\right)+1$. Desta forma podemos assumir que $A$ é finitamente gerada.

Se $V$ é um subespaço gerador de dimensão finita de $A$ que contém 1 , então $W:=V+\mathbb{K} x$ é um subespaço gerador de dimensão finita para $B=A[x ; \delta]$. Os espaços $V^{n}$ formam uma filtração exaustiva de $A$; $\log o \delta(V) \subseteq V^{m}$ para algum inteiro $m>0$, e consequentemente $\delta\left(V^{q}\right) \subseteq V^{m+q}$ para todo $q=1,2, \cdots$. Afirmamos que

$$
W^{n}=(V+\mathbb{K} x)^{n} \subseteq V^{m n}+V^{m n} x+V^{m n} x^{2}+\ldots+V^{m n} x^{n},
$$

e portanto que $d_{W}(n) \leq(n+1) d_{V}(m n)$, para todo número natural $n$. Isto é trivial para $n=0$; então vamos assumir que vale para $n \geq 0$. Logo,

$$
V W^{n} \subseteq \sum_{i=0}^{n} V^{m n+1} x^{i} \subseteq \sum_{i=0}^{n} V^{m(n+1)} x^{i}
$$

e

$$
x W^{n} \subseteq \sum_{i=0}^{n} x V^{m n} x^{i} \subseteq \sum_{i=0}^{n} V^{m n} x^{i+1}+\sum_{i=0}^{n} \delta\left(V^{m n}\right) x^{i} .
$$

Uma vez que

$$
\begin{aligned}
\delta\left(V^{m n}\right) & \subseteq \sum_{j=0}^{n} V^{j} \delta(V) V^{m n-j-1} \subseteq \sum_{j=0}^{m n-1} V^{j} V^{m} V^{m n-j-1} \\
& =V^{m(n+1)-1} \subseteq V^{m(n+1)}
\end{aligned}
$$


temos, por 2.2 e 2.3 , que

$$
x W^{n} \subseteq \sum_{i=0}^{n+1} V^{m(n+1)} x^{i}
$$

Por 2.1 e 2.4, segue que

$$
W^{n+1}=(V+\mathbb{K} x) W^{n}=V W^{n}+x W^{n} \subseteq \sum_{i=0}^{n+1} V^{m(n+1)} x^{i},
$$

completando o passo indutivo. Portanto,

$$
\begin{aligned}
G K \operatorname{dim}(B) & =\overline{\lim } \log _{n} d_{W}(n) \leq \overline{\lim } \log _{n}\left((n+1) d_{V}(m n)\right) \\
& =\lim _{n \rightarrow \infty} \log _{n}(n+1)+\overline{\lim } \log _{n} d_{V}(n)=1+\operatorname{GKdim}(A)
\end{aligned}
$$

Exemplo 2.1.7. Seja $A$ uma $\mathbb{K}$-álgebra e seja $B=A\left[x_{1}, \cdots, x_{n}\right]$. Então $G K \operatorname{dim}(B)=G K \operatorname{dim}(A)+n$, aplicando repetidamente a Proposição 2.1.6 no caso em que a derivação é nula.

Exemplo 2.1.8. Seja $A=\mathbb{R}[[x]]$ a $\mathbb{R}$-álgebra formada por todas as séries de potências com coeficientes reais. Seja $\left\{r_{i}, i=1,2, \cdots\right\}$ um conjunto infinito enumerável de números reais linearmente independentes sobre o corpo $\mathbb{Q}$. Então o conjunto das funções $\left\{f_{i}(x)=e^{r_{i} x}, i=1,2, \cdots\right\}$ é algebricamente independente sobre $\mathbb{R}$. Cada uma das funções $f_{i}$ pode ser vista como um elemento de A através de sua série de McLaurin. Logo A possui uma subálgebra isomorfa à álgebra polinomial $\mathbb{R}\left[x_{1}, \cdots, x_{n}\right]$ para cada $n \in \mathbb{N}$. Segue do Lema 2.1.1 e do Exemplo 2.1.7 que $G K \operatorname{dim}(A)=\infty$.

Observação 2.1.9. A desigualdade no Lema 2.1.5 pode, de fato, ocorrer, como mostra o resultado abaixo.

Proposição 2.1.10. ([4], página 27) Seja p um inteiro positivo.

- Existe uma $\mathbb{K}$-álgebra localmente de dimensão finita $A_{p}$ com $\mathbb{K}$-derivação $\delta_{p}$, tal que $\operatorname{GKdim}\left(A_{p}\left[t ; \delta_{p}\right]\right)=p$.

- Existe uma $\mathbb{K}$-álgebra localmente de dimensão finita $A$ com $\mathbb{K}$-derivação $\delta$, tal que $\operatorname{GKdim}(A[t ; \delta])=\infty$. 


\subsection{A álgebra de Weyl e sua dimensão de Gelfand-Kirillov}

O objetivo desta seção é calcular a dimensão de Gelfand-Kirillov da álgebra de Weyl $A_{n}$. No que se segue, vamos definir tal álgebra.

Definição 2.2.1. A álgebra de Weyl $A_{n}=A_{n}(\mathbb{K})$ é o anel de polinômios em $2 n$ variáveis $x_{1}, \cdots, x_{n}, y_{1}, \cdots, y_{n}$ com coeficientes em $\mathbb{K}$, sujeitas às relações

$$
x_{i} x_{j}=x_{j} x_{i}, \quad y_{i} y_{j}=y_{j} y_{i}, \quad \text { e } \quad x_{i} y_{j}-y_{j} x_{i}=\delta_{i j},
$$

onde $\delta_{i j}$ é o símbolo de Kronecker.

Proposição 2.2.2. Vale o isomorfismo abaixo:

$$
A_{n+1} \cong A_{n}\left[y_{n+1}\right]\left[x_{n+1} ; \delta\right], \quad \text { onde } \quad \delta=\frac{\partial}{\partial y_{n+1}}
$$

Demonstração: Primeiramente, como $y_{n+1}$ comuta com os elementos de $A_{n}$, resta mostrar que $x_{n+1}$ satisfaz as relações que definem a álgebra de Weyl. De fato, para $i=1, \cdots, n$, temos

$$
x_{i} x_{n+1}-x_{n+1} x_{i}=\delta\left(x_{i}\right)=0
$$

e

$$
y_{i} x_{n+1}-x_{n+1} y_{i}=\delta\left(y_{i}\right)=0 .
$$

Para $i=n+1$, temos

$$
y_{n+1} x_{n+1}-x_{n+1} y_{n+1}=\delta\left(y_{n+1}\right)=1,
$$

provando o resultado.

Teorema 2.2.3. A dimensão de Gelfand-Kirillov da n-ésima álgebra de Weyl $A_{n}$ é $2 n$. 
Demonstração: O resultado é provado por indução. Para $n=0$, o resultado é válido pois $A_{0}(\mathbb{K})=\mathbb{K}$.

Suponhamos que a dimensão de $A_{k}=2 k$. Pelo Exemplo 2.1.7

$$
\operatorname{GKdim}\left(A_{n}\left[y_{n+1}\right]\right)=G K \operatorname{dim}\left(A_{n}\right)+1=2 n+1
$$

Da Proposição 2.1.6, decorre que $\operatorname{GK\operatorname {dim}}\left(A_{n+1}\right)=\operatorname{GK\operatorname {dim}}\left(A_{n}\left[y_{n+1}\right]\right)+1=2(n+1)$.

\subsection{A álgebra de Weyl como o anel de operadores diferenciais do anel de polinômios $\mathbb{K}\left[x_{1}, \cdots, x_{n}\right]$}

Uma outra definição da álgebra de Weyl é encontrada com frequência. Por conveniência, denotaremos por $\mathbb{K}[X]$ o anel $\mathbb{K}\left[x_{1}, \cdots, x_{n}\right]$.

Definição 2.3.1. Sejam $\hat{x}_{1}, \cdots, \hat{x}_{n}$ os operadores de $\mathbb{K}[X]$ definidos sobre um polinômio $f \in \mathbb{K}[X]$ pelas fórmulas $\hat{x}_{i}(f)=x_{i} . f$. Analogamente, $\partial_{1}, \cdots, \partial_{n}$ são os operadores definidos por $\partial_{i}=\partial f / \partial x_{i}$. Estes são operadores lineares de $\mathbb{K}[X]$. A n-ésima álgebra de Weyl $A_{n}$ é a $\mathbb{K}$-subálgebra de $\operatorname{End}_{\mathbb{K}}(\mathbb{K}[X])$ gerada pelos operadores $\hat{x}_{1}, \cdots, \hat{x}_{n}$ e $\partial_{1}, \cdots, \partial_{n}$.

De acordo com a definição acima, os elementos de $A_{n}$ são combinações lineares sobre $\mathbb{K}$ de monômios em $\hat{x}_{1}, \cdots, \hat{x}_{n}, \partial_{1}, \cdots, \partial_{n}$. Porém, vale ressaltar que esta álgebra não é comutativa. De fato, consideremos o operador $\partial_{i} . \hat{x}_{i}$ e vamos aplicá-lo no polinômio $f \in \mathbb{K}[X]$. Usando a regra de derivação do produto, obtemos $\partial_{i} . \hat{x}_{i}(f)=x_{i} \partial f / \partial x_{i}+f$. Em outras palavras,

$$
\partial_{i} \cdot \hat{x}_{i}=\hat{x}_{i} \cdot \partial_{i}+1
$$

onde 1 corresponde ao operador identidade. Utilizando a notação de comutadores, a expressão acima se torna $\left[\partial_{i}, \hat{x}_{i}\right]=1$. Através de cálculos similares obtemos que $\left[\partial_{i}, \hat{x}_{j}\right]=\delta_{i j} .1$ e $\left[\partial_{i}, \partial_{j}\right]=\left[\hat{x}_{i}, \hat{x}_{j}\right]=0$, onde mais uma vez $\delta_{i j}$ é o símbolo de Kronecker, e $1 \leq i, j \leq n$. Denotaremos abaixo os operadores $\hat{x}_{i}$ simplesmente por $x_{i}$ para simplificar a notação.

O primeiro passo para mostrar a equivalência destas definições é construir uma base para a álgebra de Weyl como $\mathbb{K}$-espaço vetorial, a qual chamaremos base canônica. Ela será 
descrita através de multi-índices, ou seja, elementos $\alpha \in \mathbb{N}^{n}$ tais que $\alpha=\left(\alpha_{1}, \ldots, \alpha_{n}\right)$. Por $x^{\alpha}$ denotaremos o monômio $x_{1}^{\alpha_{1}} \cdots x_{n}^{\alpha_{n}}$. O grau deste monômio é o comprimento $|\alpha|$ do multi-índice $\alpha$, ou seja, $|\alpha|=\alpha_{1}+\cdots+\alpha_{n}$. Também definimos o fatorial de um multi-índice $\beta \in \mathbb{N}^{n}$ por $\beta !=\beta_{1} ! \cdots \beta_{n}$ !

Lema 2.3.2. Sejam $\alpha, \beta \in \mathbb{N}^{n}$ e suponhamos que $|\alpha| \leq|\beta|$. Então $\partial^{\beta}\left(x^{\alpha}\right)=\beta$ ! se $\alpha=\beta$ e zero, caso contrário.

Demonstração: Se $\alpha=\beta$, o resultado decorre da regra do produto, uma vez que $\partial_{i}^{\alpha_{i}}\left(x_{i}^{\alpha_{i}}\right)=\alpha_{i}$ !. Se $\alpha \neq \beta$, existe $1 \leq n_{0} \leq n$ tal que $\alpha_{n_{0}}<\beta_{n_{0}}$. Como $\partial^{\beta_{n_{0}}}\left(x^{\alpha_{n_{0}}}\right)=0$, segue que $\partial^{\beta}\left(x^{\alpha}\right)=0$.

Lema 2.3.3. Seja $f \in \mathbb{K}[X]$. Então $\left[\partial_{i}, f\right]=\partial f / \partial x_{i}$.

Demonstração: Pelas relações apresentadas acima e pelo fato de o comutador ser linear, é suficiente mostrar que $\left[\partial_{i}, x_{i}^{n}\right]=n x_{i}^{n-1}$. Para $n=0$, o resultado é trivial. Suponhamos que a identidade seja válida para $n=k$. Como, dados $a, b, c \in A_{n},[a, b c]=b[a, c]+[a, b] c$, temos que

$$
\left[\partial_{i}, x_{i}^{k+1}\right]=x_{i}\left[\partial_{i}, x_{i}^{k}\right]+\left[\partial_{i}, x_{i}\right] x_{i}^{k}=x_{i} \cdot k \cdot x_{i}^{k-1}+x_{i}^{k}=(k+1) x_{i}^{k} .
$$

Proposição 2.3.4. O conjunto $B=\left\{x^{\alpha} \partial^{\beta}, \alpha, \beta \in \mathbb{N}^{n}\right\}$ é uma base de $A_{n}$ como espaço vetorial sobre $\mathbb{K}$.

Demonstração: Dado um monômio de $A_{n}$, pelo Lema 2.3.3 é possível escrevê-lo como uma soma de monômios com potências dos operadores $x_{i}$ à esquerda e potências dos operadores $\partial_{i}$ à direita. Desta forma, segue que qualquer elemento de $A_{n}$ pode ser escrito como combinação linear de elementos de $B$.

Agora mostremos que os elementos de $B$ são linearmente independentes. Consideremos uma combinação linear finita $D=\sum c_{\alpha \beta} x^{\alpha} \partial^{\beta}$ de elementos de $B$. Queremos mostrar que se algum $c_{\alpha \beta} \neq 0$, então $D \neq 0$. Mas $D$ é um operador linear em $\mathbb{K}[X]$. Portanto $D \neq 0$ se, e somente se, existe um polinômio $f$ tal que $D(f) \neq 0$. Construiremos tal $f$. 
Seja $\sigma$ um multi-índice que satisfaz $c_{\alpha \sigma} \neq 0$ para algum índice $\alpha$, mas $c_{\alpha \beta}=0$ para todo índice $\beta$ tal que $|\beta|<|\sigma|$. Pelo Lema 2.3.2, temos que $D\left(x^{\sigma}\right)=\sigma ! \sum_{\alpha} c_{\alpha \sigma} x^{\alpha} \neq 0$, pois um dos $c_{\alpha \sigma}$ é diferente de zero, pela escolha de $\sigma$. Logo $f=x^{\sigma}$ é o polinômio procurado.

Agora vamos mostrar que as duas definições são equivalentes. Seja $\mathbb{K}\left\langle z_{1}, \cdots, z_{2 n}\right\rangle$ a álgebra livre em $2 n$ geradores, ou seja, o conjunto de todas as combinações lineares finitas de palavras em $z_{1}, \ldots, z_{2 n}$. Podemos definir um homomorfismo sobrejetor $\phi: \mathbb{K}\left\langle z_{1}, \cdots, z_{2 n}\right\rangle \rightarrow A_{n}$ tal que $\phi\left(z_{i}\right)=x_{i}$ e $\phi\left(z_{i+n}\right)=\partial_{i}$, para $i=1, \ldots, n$. Seja $J$ o ideal bilateral gerado por $\left[z_{i+n}, z_{i}\right]-1$, para $i=1,2, \cdots, n$ e $\left[z_{i}, z_{j}\right]$ para $j \neq i+n$ e $1 \leq i, j \leq n$ Segue das relações do início desta seção que $J \subseteq \operatorname{Ker}(\phi)$. Logo $\phi$ induz um homomorfismo de $\mathbb{K}$-álgebras $\hat{\phi}: \mathbb{K}\left\langle z_{1}, \cdots, z_{2 n}\right\rangle / J \rightarrow A_{n}$.

Teorema 2.3.5. A aplicação

$$
\hat{\phi}: \mathbb{K}\left\langle z_{1}, \cdots, z_{2 n}\right\rangle / J \rightarrow A_{n}
$$

é um isomorfismo de $\mathbb{K}$-álgebras.

Demonstração: Assim como na prova da Proposição 2.3.4, podemos usar as relações entre as classes $z_{i}+J$ para mostrar que todo elemento de $\mathbb{K}\left\langle z_{1}, \cdots, z_{2 n}\right\rangle$ pode ser escrito como uma combinação linear de monômios do tipo $z_{1}^{m_{1}} \cdots z_{2 n}^{m_{2 n}}$. Pela Proposição 2.3.4, a imagem desses monômios por $\hat{\phi}$ é uma base de $A_{n}$ como espaço vetorial sobre $\mathbb{K}$. Em particular, os monômios são linearmente independentes em $\mathbb{K}\left\langle z_{1}, \cdots, z_{2 n}\right\rangle / J$. Portanto $\hat{\phi}$ é um isomorfismo de espaços vetoriais, uma vez que já é um isomorfismo de anéis. 


\section{CAPÍTULO 3}

\section{MÓDULOS E ÁLGEBRAS FILTRADOS E GRADUADOS}

A dimensão clássica de Krull, definida em termos de cadeias descendentes de ideais primos, não é uma ferramenta muito adequada no estudo de anéis não comutativos, principalmente por não se aplicar a módulos. Por outro lado, a dimensão de Gelfand-Kirillov pode ser estendida a módulos. Um primeiro estudo dessa extensão foi feito por Bernstein e, posteriormente, um estudo sistemático foi feito por Joseph e Small. Neste capítulo definiremos a dimensão de Gelfand-Kirillov para módulos e apresentaremos os resultados básicos sobre módulos filtrados e graduados.

\subsection{A dimensão de Gelfand-Kirillov de um módulo}

Seja $A$ uma $\mathbb{K}$-álgebra finitamente gerada com subespaço gerador de dimensão finita $V$ que contém 1, e seja $M$ um $A$-módulo à direita finitamente gerado com um espaço vetorial $F$ de dimensão finita que gera $M$ como $A$-módulo. Então

$$
M=\bigcup_{n=0}^{\infty} F V^{n} .
$$


Assim como no Lema 1.1.2 é possível verificar que o crescimento $\mathcal{G}\left(d_{V, F}\right)$ da função $d_{V, F}(n)=\operatorname{dim}_{\mathbb{K}}\left(F V^{n}\right)$ não depende das escolhas particulares para os espaços $V$ e $F$; portanto nos referimos ao crescimento $\mathcal{G}(M)$ do módulo $M$, e definimos

$$
\operatorname{GKdim}(M)=\overline{\lim } \log _{n} d_{V, F}(n)
$$

Mais geralmente, definimos

Definição 3.1.1. Seja A uma $\mathbb{K}$-álgebra, e seja $M$ um A-módulo à direita. A dimensão de Gelfand-Kirillov de $M$ é dada por

$$
G K \operatorname{dim}(M)=\sup _{V, F} \overline{l i m} \log _{n} \operatorname{dim}_{\mathbb{K}}\left(F V^{n}\right)
$$

onde o supremo é tomado sobre todos os subespaços $V$ de dimensão finita de A que contém 1 e todos os subespaços $F$ de dimensão finita de $M$. Alternativamente,

$$
G K \operatorname{dim}\left(M_{A}\right)=\sup _{B, N} G K \operatorname{dim}\left(N_{B}\right),
$$

onde o supremo é tomado sobre todas as subálgebras $B$ de A finitamente geradas e todos os $B$-submódulos à direita $N$ de $M$ finitamente gerados. Notemos que $G K \operatorname{dim}(0)=-\infty$

Segue da definição que $G K \operatorname{dim}(A)$, a dimensão de Gelfand-Kirillov de $A$ como álgebra , e $G K \operatorname{dim}\left(A_{A}\right)$, a dimensão de $A$ como $A$-módulo à direita, coincidem.

Lema 3.1.2. Se B é submódulo ou imagem homomorfa de um módulo $A$, então $G K \operatorname{dim}(B) \leq$ $\operatorname{GKdim}(A)$.

Demonstração: Para submódulos o resultado é imediato. Para um módulo quociente $\bar{M}$ de $M$ consideramos subespaços $V$ de $A$ e $\bar{F}$ de $\bar{M}$ de dimensão finita. A partir dos elementos da base de $\bar{F}$ podemos obter, em $M$, um conjunto de representantes, os quais formam uma base de um subespaço $F$ de $M$, de dimensão finita. Assim, temos que $\operatorname{dim}\left(\bar{F} V^{n}\right) \leq\left(\operatorname{dim} F V^{n}\right)$, para todo $n \in \mathbb{N}$. Então

$$
\overline{\lim } \log _{n} \operatorname{dim}\left(\bar{F} V^{n}\right) \leq \overline{\lim } \log _{n} \operatorname{dim}\left(F V^{n}\right)
$$

e portanto, 


$$
\sup _{V, \bar{F}} \overline{\lim } \log _{n} \operatorname{dim}\left(\bar{F} V^{n}\right) \leq \sup _{V, F} \overline{\lim } \log _{n} \operatorname{dim}\left(F V^{n}\right)
$$

Proposição 3.1.3. Seja A uma $\mathbb{K}$-álgebra, e seja $M$ um A-módulo à direita. Então

(a) Se $M=\oplus_{i=1}^{n} M_{i}$, então $G K \operatorname{dim}(M)=\max _{i}\left\{G K \operatorname{dim}\left(M_{i}\right)\right\}$.

(b) $S e 0 \rightarrow K \stackrel{\psi}{\rightarrow} M \stackrel{\phi}{\rightarrow} L \rightarrow 0$ é uma sequência exata de A-módulos à direita, então $\operatorname{GKdim}(M) \geq \max \{G K \operatorname{dim}(K), G K \operatorname{dim}(L)\}$.

(c) Se $M I=0$ para um ideal I de A, então $G K \operatorname{dim}\left(M_{A}\right)=G K \operatorname{dim}\left(M_{A / I}\right)$.

(d) $\operatorname{GKdim}\left(M_{A}\right) \leq G \operatorname{Kdim}(A)$.

(e) Se $M$ é finitamente gerado e $\alpha \in \operatorname{End}_{A}(M)$ é injetor, então

$$
\operatorname{GKdim}(M / \alpha(M)) \leq G K \operatorname{dim}(M)-1
$$

Demonstração: (a) Da definição da dimensão de Gelfand-Kirillov para módulos e do Lema 3.1 .2 , temos que

$$
\gamma:=\max \left\{\operatorname{GKdim}\left(M_{i}\right)\right\} \leq G K \operatorname{dim}\left(\oplus_{i=1}^{n} M_{i}\right),
$$

e a igualdade vale se $G K \operatorname{dim}\left(M_{i}\right)=\infty$ para algum $M_{i}$. Suponhamos que $\gamma$ é finito e $n=2$, sem perda de generalidade.

Seja $F W$ tal que $F=F_{1} \oplus F_{2}$ é subespaço de $M$, onde $F_{1}$ e $F_{2}$ geram $M_{1}$ e $M_{2}$, respectivamente, e ambos são de dimensão finita, e $W$ subespaço de $A$ de dimensão finita que contém 1. Temos

$$
F=F_{1} \oplus F_{2} \Rightarrow F V=\left(F_{1} \oplus F_{2}\right) V=F_{1} V \oplus F_{2} V
$$


Pelo Lema 1.2.1, temos

$$
d_{V, F_{1}}(n) \leq n^{\gamma+\frac{\epsilon}{2}} \quad e \quad d_{V, F_{2}}(n) \leq n^{\gamma+\frac{\epsilon}{2}}
$$

para quase todo $n$. Como $n^{\frac{\epsilon}{2}}>2$ para algum $n$ suficientemente grande, temos

$$
d_{V, F}(n)=d_{V, F_{1}}(n)+d_{V, F_{2}}(n) \leq 2 n^{\gamma+\frac{\epsilon}{2}}<n^{\frac{\epsilon}{2}} n^{\gamma+\frac{\epsilon}{2}}=n^{\gamma+\epsilon}
$$

para quase todo $n$. Então $\overline{l i m} \log _{n} d_{V, F} \leq \gamma$ pelo Lema 1.2.1, e portanto

$$
\operatorname{GKdim}\left(M_{1} \oplus M_{2}\right)=\sup \overline{\lim } \log _{n} d_{W}(n) \leq \gamma
$$

(b) Como $K \cong \psi(K)$ (pois é injetora) e $\operatorname{dim}_{\mathbb{K}}(K) \leq \operatorname{dim}_{\mathbb{K}}(M)$, segue que $G K \operatorname{dim}(K) \leq$ $G K \operatorname{dim}(M)$.

Quanto à outra desigualdade, como $\phi$ é sobrejetora, sabemos que $L \cong M / \operatorname{Ker} \phi$. Logo, pelo 3.1.2, $G K \operatorname{dim}(L) \leq G K \operatorname{dim}(M)$.

(c) Seja $F$ subespaço de $M, V$ subespaço gerador de $A$ com unidade. Como $M I=0, M$ pode ser visto como um $A / I$-módulo tal que $m \cdot \bar{a}:=m a$.

Seja $\bar{V}$ o conjunto formado pelos elementos correspondentes de $V$ em $A / I$. Então

$$
\operatorname{dim}\left(F V^{n}\right)=\operatorname{dim}\left(F \bar{V}^{n}\right)
$$

Consequentemente

$$
\overline{\lim } \log _{n} \operatorname{dim}\left(F V^{n}\right)=\overline{\lim } \log _{n} \operatorname{dim}\left(F \bar{V}^{n}\right)
$$

Então

$\overline{\lim } \log _{n} \operatorname{dim}\left(F V^{n}\right) \leq \sup _{F, W} \overline{\lim } \log _{n} \operatorname{dim}\left(F \bar{W}^{n}\right) \Rightarrow \sup _{F, V} \overline{\lim } \log _{n} \operatorname{dim}\left(F V^{n}\right) \leq \sup _{F, W} \overline{\lim } \log _{n} \operatorname{dim}\left(F \bar{W}^{n}\right)$, onde o supremo é tomado sobre todos os subespaços geradores com unidade $W$ de $A / I$.

A desigualdade inversa é obtida de maneira análoga a partir da igualdade acima.

(d) Seja $F$ um subespaço de dimensão finita de $M$, e seja $V$ um subespaço de dimensão finita de $A$ que contém 1. Claramente, $\operatorname{dim}_{\mathbb{K}}\left(F V^{n}\right) \leq \operatorname{dim}_{\mathbb{K}}(F) \operatorname{dim}_{\mathbb{K}}\left(V^{n}\right)$. Logo $\mathcal{G}\left(d_{F, V}\right) \leq$ $\mathcal{G}\left(d_{V}\right)$. Portanto $G K \operatorname{dim}\left(M_{A}\right) \leq G \operatorname{Kdim}(A)$. 
(e) Seja $V$ um subespaço de dimensão finita de $A$, com unidade, e seja $\bar{F}$ um subespaço de dimensão finita de $\bar{M}=M / \alpha(M)$ que gera $\bar{M}$ como $A$-módulo. Como $\alpha(M)$ também é finitamente gerado, existe um subespaço $F$ de $M$ de dimensão finita tal que $F$ gera $M$ como $A$-módulo e $\bar{F}$ é a imagem de $F$ sob o homomorfismo canônico $M \rightarrow M / \alpha(M)$.

Para cada inteiro $n>0$, seja $C_{n}$ o espaço vetorial complementar a $\alpha(M) \cap F V^{n}$ em $F V^{n}$; $\operatorname{logo} C_{n} \cong \bar{F} V^{n}$. Como $C_{n} \cap \alpha(M)=0$, a soma

$$
C_{n}+\alpha\left(C_{n}\right)+\alpha^{2}\left(C_{n}\right)+\cdots+\alpha^{j}\left(C_{n}\right)
$$

de subespaços é direta para todo $j$. De fato, se

$$
a_{0} x_{0}+a_{1} \alpha\left(x_{1}\right)+\cdots+a_{j} \alpha^{j}\left(x_{j}\right)=0,
$$

onde $x_{i} \in C_{n}$ e $a_{i} \in \mathbb{K}, 0 \leq i \leq j$, temos

$$
\alpha\left(a_{1} x_{1}+\cdots+a_{j} \alpha^{j-1}\left(x_{j}\right)\right)=-a_{0} x_{0} .
$$

Como $-a_{0} x_{0}$ está em $C_{n}$ e em $\alpha(M)$, se $a_{0} \neq 0$, segue que $x_{0}=0$. Como $\alpha$ é injetor, isto implica que $a_{1} x_{1}+\cdots+a_{j} \alpha^{j-1}\left(x_{j}\right)=0$. Aplicando este processo recursivamente, temos que $a_{i} \alpha^{i}\left(x_{i}\right)=0$ para todo $i$, provando que a soma é direta.

Como $\alpha(F)$ tem dimensão finita e $F$ gera $M$ como $A$-módulo, existe um subespaço de dimensão finita $W \supseteq V$ de $A$ tal que $\alpha(F) \subseteq F W$.

Assim,

$$
\bigoplus_{j=0}^{n} \alpha^{j}\left(C^{n}\right) \subseteq \bigoplus_{j=0}^{n} \alpha^{j}\left(F V^{n}\right)=\bigoplus_{j=0}^{n} \alpha^{j}(F) V^{n} \subseteq \bigoplus_{j=0}^{n} F W^{j} W^{n} \subseteq F W^{2 n},
$$

e consequentemente

$$
\operatorname{dim}_{\mathbb{K}}\left(F W^{2 n}\right) \geq(n+1) \operatorname{dim}_{\mathbb{K}}\left(C_{n}\right)=(n+1) \operatorname{dim}_{\mathbb{K}}\left(\bar{F} V^{n}\right)
$$

de onde segue o resultado.

Em geral, a parte (e) do teorema acima não é válida se $M$ não é um $A$-módulo à direita finitamente gerado. Se $M=\mathbb{K}[x]$ é considerado como um $\mathbb{K}$-módulo à direita, então $\operatorname{GK\operatorname {dim}}\left(M_{\mathbb{K}}\right)=0$. Notemos que a multiplicação à direita por $x$ induz um $\mathbb{K}$-homomorfismo $\alpha$ de $M$. Então 


$$
\operatorname{GKdim}(M / \alpha(M))=0=G K \operatorname{dim}(M),
$$

uma vez que $M / \alpha(M) \cong \mathbb{K}$.

Há situações onde a desigualdade em (b) pode ser uma igualdade, o que motiva a próxima definição.

Definição 3.1.4. Seja A uma $\mathbb{K}$-álgebra. A dimensão de Gelfand-Kirillov é exata para A-módulos à direita se

$$
G K \operatorname{dim}(M)=\max \{G K \operatorname{dim}(L), G K \operatorname{dim}(N)\}
$$

para qualquer sequência exata curta $0 \rightarrow L \rightarrow M \rightarrow N \rightarrow 0$ de A-módulos à direita.

\section{2 Álgebras filtradas e graduadas}

Frequentemente, as álgebras são munidas de uma filtração natural, e informações podem ser obtidas ao passar para a álgebra graduada associada, e então trazendo os resultados de volta para a álgebra inicial. Isto é particularmente útil se a álgebra é filtrada por subespaços de dimensão finita tais que a álgebra graduada associada é comutativa - esse tipo de álgebra é chamado álgebra quase-comutativa. A dimensão de Gelfand-Kirillov é uma ferramenta efetiva no seu estudo, o qual será feito no capítulo seguinte.

Apesar de graduações gerais já terem sido estudadas, nos preocuparemos com graduações cujas componentes estão indexadas por números naturais ou inteiros. Além disso, nos restringiremos às graduações por subespaços de dimensão finita, as chamadas graduações finitas.

Definição 3.2.1. Uma graduação $\mathcal{A}=\left\{A_{i}\right\}_{i \in \mathbb{Z}} d a \mathbb{K}$-álgebra $A$ é uma sequência de $\mathbb{K}$ subespaços $A_{i}$ de $A$ tais que

$$
A=\bigoplus_{i \in \mathbb{Z}} A_{i} \quad \text { e } \quad A_{i} \cdot A_{j} \subseteq A_{i+j} \quad \text { para todo } \quad i, j \in \mathbb{Z} .
$$

Uma álgebra com uma graduação $\mathcal{A}$ é chamada $\mathcal{A}$-graduada, ou simplesmente graduada; é finitamente graduada se cada uma das componentes $A_{i}$ é um $\mathbb{K}$-espaço vetorial de dimensão finita. Os elementos de $A_{n}$ são ditos homogêneos de grau $n$. A componente $A_{0}$ 
é uma subálgebra de A que contém $1_{A}$ e portanto contém o corpo base $\mathbb{K}$. Se A é finitamente graduada, $A_{0}=\mathbb{K}$, e $A_{i}=0$ para todo $i<0$, então A é chamado graduada conexa.

Definição 3.2.2. Seja A uma $\mathbb{K}$-álgebra com graduação $\mathcal{A}=\left\{A_{i}\right\}_{i \in \mathbb{Z}}$. Um A-módulo à direita $M$ é $\mathcal{A}$-graduado, ou simplesmente graduado, se existem subespaços $M_{i}$ tais que

$$
M=\bigoplus_{i \in \mathbb{Z}} M_{i} \quad \text { e } \quad M_{i} A_{j} \subseteq M_{i+j} \quad \text { para todo } \quad i, j \in \mathbb{Z} .
$$

Se cada $M_{i}$ possui dimensão finita sobre $\mathbb{K}$, então $M$ é dito finitamente graduado. Os elementos de $M_{n}$ são chamados homogêneos de grau $n$.

Seja $A=\oplus_{i \in \mathbb{Z}} A_{i}$ uma $\mathbb{K}$-álgebra finitamente graduada, e seja $M=\oplus_{i \in \mathbb{Z}} M_{i}$ um $A$-módulo finitamente graduado. Defina

$$
\begin{aligned}
& A(n)=\bigoplus_{i=-n}^{n} A_{i}, \quad d_{A}(n)=\operatorname{dim}_{\mathbb{K}} A(n), \quad \mathrm{e} \\
& M(n)=\bigoplus_{i=-n}^{n} M_{i}, \quad d_{M}(n)=\operatorname{dim}_{\mathbb{K}} M(n) .
\end{aligned}
$$

O lema seguinte mostra que $\mathcal{G}\left(d_{M}\right)$ fornece um limitante superior para o crescimento do módulo $M$ e que a igualdade vale se $A$ é uma álgebra finitamente gerada e $M$ é um $A$-módulo finitamente gerado à direita.

Lema 3.2.3. Seja A uma $\mathbb{K}$-álgebra finitamente graduada, e seja $M$ um A-módulo finitamente graduado. Então

(a) Se $V$ é um subespaço de dimensão finita de A que contém 1 e E é um subespaço de dimensão finita de $M$, então $\mathcal{G}\left(d_{V, E}\right) \leq \mathcal{G}\left(d_{M}\right)$, e portanto $G K \operatorname{dim}(M) \leq \overline{\lim } \log _{n} d_{M}(n)$.

(b) Se A é finitamente gerado como álgebra e $M_{A}$ é finitamente gerado, então $\mathcal{G}(M)=\mathcal{G}\left(d_{M}\right)$ e portanto $G K \operatorname{dim}(M)=\overline{\lim } \log _{n} d_{M}(n)$.

Demonstração: (a) Como $V$ e $E$ possuem dimensão finita, existe um número natural $p$ tal que $V \subseteq A(p)$ e $E \subseteq M(p)$. Portanto

$$
E V^{n} \subseteq E A(n p) \subseteq M(n p+p) \subseteq M(2 p n)
$$


para qualquer inteiro $n \geq 1$. Consequentemente, $d_{V, E}(n) \leq d_{M}(2 p n)$, e segue a afirmação.

(b) Para $p$ inteiro suficientemente grande, o espaço vetorial $V=A(p)$ gera $A$ e contém 1, e $E=M(p)$ gera $M$ como $A$-módulo. Queremos mostrar que $M(n) \subseteq E V^{n}$ para todo $n>0$, e é suficiente mostrar que $M_{-n}+M_{n} \subseteq E V^{n}$. Demonstraremos que $M_{n} \subseteq E V^{n}$; um argumento simétrico nos dá que $M_{-n} \subseteq E V^{-n}$. Como os subespaços $E V^{m}, m=0,1, \cdots$, fornecem uma filtração exaustiva de $M, M_{n} \subseteq E V^{r}$ para algum inteiro positivo $r$. Cada elemento $0 \neq x \in M_{n}$ é, portanto, uma soma de elementos não nulos $v_{0} v_{1} \cdots v_{s}$ com elementos homogêneos

$$
v_{0} \in E, v_{i} \in V, 1 \leq i \leq s, \quad \text { onde } \quad s<r .
$$

Vamos assumir que cada monômio foi simplificado ao máximo, isto é, assumiremos que

$$
v_{0} v_{1} \notin E=M(p) \quad \text { e } \quad v_{i} v_{i+1} \notin V=A(p) \quad \text { para } \quad i \geq 1
$$

ou, equivalentemente, que $\left|\operatorname{deg}\left(v_{i} v_{i+1}\right)\right|>p$, para $i \geq 0$. Como

$$
\operatorname{deg}\left(v_{0} v_{1} \cdots v_{s}\right)=\operatorname{deg}\left(v_{0}\right)+\operatorname{deg}\left(v_{1}\right)+\cdots+\operatorname{deg}\left(v_{s}\right)=n>0,
$$

pelo menos um dos $v_{i}$ deve ter grau positivo. Suponhamos que $\operatorname{deg}\left(v_{i}\right)>0, \operatorname{mas} \operatorname{deg}\left(v_{i+1}\right) \leq 0$ para $\operatorname{algum} i$. Então

$$
\left|\operatorname{deg}\left(v_{i} v_{i+1}\right)\right| \leq \max \left\{\left|\operatorname{deg}\left(v_{i}\right)\right|,\left|\operatorname{deg}\left(v_{i+1}\right)\right|\right\} \leq p,
$$

uma contradição (para $\mathrm{i}=\mathrm{s}$, considerar $v_{s-1} v_{s}$ ). Portanto todo $v_{i}$ possui grau positivo, logo $n \geq s+1$ e consequentemente $v_{0} v_{1} \cdots v_{s} \in E V^{n}$, logo $M_{n} \subseteq E V^{n}$.

Definição 3.2.4. Uma $\mathbb{Z}$-filtração, ou simplesmente uma filtração de uma $\mathbb{K}$-álgebra $A$ é uma sequência de $\mathbb{K}$-subespaços

$$
\cdots \subseteq A_{i-1} \subseteq A_{i} \subseteq A_{i+1} \subseteq \cdots, i \in \mathbb{Z}
$$

tal que

$$
1 \in A_{0}, \quad A_{i} \cdot A_{j} \subseteq A_{i+j} \quad \text { para todo } \quad i, j \in \mathbb{Z}, \quad \text { e } \quad A=\bigcup_{i \in \mathbb{Z}} A_{i}
$$


Uma filtração é chamada finita se cada $A_{i}$ tem dimensão finita sobre $\mathbb{K}$, e é chamada discreta se $A_{i}=0$ para todo $i<n_{0}$ para algum inteiro $n_{0} \leq 0$. O $\mathbb{K}$-espaço vetorial

$$
\operatorname{gr}(A)=\bigoplus_{i \in \mathbb{Z}} A_{i} / A_{i-1}
$$

munido de uma multiplicação dada pela regra

$$
\left(x+A_{i-1}\right) \cdot\left(y+A_{j-1}\right)=x y+A_{i+j-1},
$$

onde $x$ e y sao elementos homogêneos de gr $(A)$, é chamado álgebra graduada associada. A operação se estende aos demais elementos de A por linearidade.

Veremos abaixo que se a álgebra graduada associada de uma $\mathbb{K}$-álgebra discretamente filtrada $A$ é finitamente gerada, então $A$ é finitamente gerado.

Suponhamos que $\operatorname{gr}(A)$ é gerado por $\bar{x}_{1}, \cdots, \bar{x}_{r}$. Podemos supor que cada um desses geradores é homogêneo, sem perda de generalidade. Desta forma, a cada $\bar{x}_{i}$ podemos associar um $x_{i} \in A$. Seja $B \subseteq A$ a subálgebra gerada por $x_{1}, \cdots, x_{r}$. Vamos mostrar que $A \subseteq B$. Por indução, mostraremos que cada $A_{n} \subseteq B, n \geq n_{0}$.' Decorre da hipótese de a filtração ser discreta que $A_{n_{0}} \subseteq B$, uma vez que a primeira parcela não nula de $g r(A)=\oplus_{-\infty}^{\infty} A_{i} / A_{i-1}$ é justamente $A_{n_{0}}$. Suponhamos agora que $A_{k} \subseteq B$, para $k \geq n_{0}$. Mostremos que $A_{k+1} \subseteq B$. Seja $y \in A_{k+1}$. Ele possui um elemento correspondente $\bar{y} \in A_{k+1} / A_{k}$. Como $\operatorname{gr}(A)$ é finitamente gerado, podemos escrever

$$
\bar{y}=\sum_{j} \alpha_{j} \bar{x}_{i_{1}} \cdots \bar{x}_{s}
$$

onde $1 \leq i_{1}, \cdots, i_{s} \leq r$. Isto significa que $y-\sum_{j} \alpha_{j} x_{i_{1}} \cdots x_{i_{s}} \in A_{k}$. Como, pela hipótese de indução, $A_{k} \subseteq B$ e $\sum_{j} \alpha_{j} x_{i_{1}} \cdots x_{i_{s}} \in B$, segue que $y \in B$.

Definição 3.2.5. Seja A uma $\mathbb{K}$-álgebra filtrada por subespaços $A_{i}, i \in \mathbb{Z}$, e seja $M$ um A-módulo à direita. Uma filtração de $M$ é uma sequência de subespaços

$$
\cdots \subseteq M_{i-1} \subseteq M_{i} \subseteq M_{i+1} \subseteq \cdots, i \in \mathbb{Z}
$$

tal que 


$$
M_{i} \cdot A_{j} \subseteq M_{i+j} \quad \text { para todo } \quad i, j \in \mathbb{Z}, \quad \text { e } \quad M=\bigcup_{i \in \mathbb{Z}} M_{i} .
$$

Uma filtração é finita se cada um dos espaços vetoriais $M_{i}$ tem dimensão finita sobre $\mathbb{K}$, e é discreta se $M_{i}=0$ para todo $i<n_{0}$ para algum inteiro $n_{0}$. O $\mathbb{K}$-espaço vetorial

$$
\operatorname{gr}(M)=\bigoplus_{i \in \mathbb{Z}} M_{i} / M_{i-1},
$$

com a estrutura de gr $(A)$-módulo dada pela regra

$$
\left(m+M_{i-1}\right) \cdot\left(a+A_{j-1}\right)=m a+M_{i+j-1},
$$

onde $m$ e a são elementos homogêneos de $\operatorname{gr}(M)$ e $\operatorname{gr}(A)$, respectivamente, é chamado módulo graduado associado. A operação se estende aos demais elementos de $M$ por linearidade.

De forma análoga à que vimos anteriormente, é possível mostrar que se o módulo graduado associado de um módulo discretamente filtrado é finitamente gerado, então o módulo original também é finitamente gerado.

Seja $A$ uma $\mathbb{K}$-álgebra com filtração $\left\{A_{i}\right\}_{i \in \mathbb{Z}}$, e seja $M$ um $A$-módulo à direita com filtração $\left\{M_{i}\right\}_{i \in \mathbb{Z}}$. Utilizando os homomorfismos canônicos

$$
g r_{i}: M_{i} \rightarrow M_{i} / M_{i-1}, \quad \text { defina } \operatorname{gr}(E)=\bigoplus_{i \in \mathbb{Z}} g r_{i}\left(E \cap M_{i}\right)
$$

para qualquer subespaço $E$ de $M$. Se $E$ é um submódulo de $M$, é fácil ver que $\operatorname{gr}(E)$ é um $\operatorname{gr}(A)$-submódulo de $\operatorname{gr}(M)_{g r(A)}$. A aplicação $E \rightarrow \operatorname{gr}(E)$ preserva a ordem entre os submódulos de $M_{A}$ e os submódulos de $\operatorname{gr}(M)_{\operatorname{gr}(A)}$. Se a filtração é discreta, então esta aplicação preserva inclusões estritas, como veremos a seguir.

Sejam $E$ e $F$ submódulos de $M_{A}$ tais que $E \subseteq F$ e $\operatorname{gr}(E)=\operatorname{gr}(F)$. Vamos assumir que $F \backslash E \neq \emptyset$ e tome $i$ minimal com relação à propriedade de que existe um elemento $f \in F \cap M_{i}, f \notin E$ - a existência deste $i$ é garantida pelo fato de a filtração ser discreta. Como $\operatorname{gr}(E)=\operatorname{gr}(F)$, temos que

$$
g r_{i}(f)=f+M_{i-1}=e+M_{i-1}=g r_{i}(e)
$$


para algum $e \in E$. Portanto, $f-e \in M_{i-1}$. Como $E \subseteq F$, segue que $f-e \in F$. Pela minimalidade de $i$, segue que $f-e \in E$, de onde decorre que $f \in E$, uma contradição. Deste fato segue o próximo resultado.

Proposição 3.2.6. Seja A uma $\mathbb{K}$-álgebra filtrada, e seja $M$ um A-módulo discretamente filtrado. Se $\operatorname{gr}(M)_{\operatorname{gr}(A)}$ é noetheriano, então $M_{A}$ é noetheriano.

Lema 3.2.7. Seja A uma $\mathbb{K}$-álgebra com uma filtração $\left\{A_{i}\right\}_{i \in \mathbb{Z}}$, e seja $M$ um A-módulo à direita filtrado com filtração $\left\{M_{i}\right\}_{i \in \mathbb{Z}}$. Então

$$
G K \operatorname{dim}\left(\operatorname{gr}(M)_{\operatorname{gr}(A)}\right) \leq G K \operatorname{dim}\left(M_{A}\right) .
$$

Demonstração: Seja $W$ um subespaço de dimensão finita de $\operatorname{gr}(A)$ que contém 1, e seja $F$ um subespaço de dimensão finita de $\operatorname{gr}(M)$. Então existe um subespaço $V$ de $A$ de dimensão finita que contém 1 e um subespaço de dimensão finita $E$ de $M$ tal que $W \subseteq g r(V)$ e $F \subseteq g r(E)$. Então

$$
F W^{n} \subseteq \operatorname{gr}(E) \operatorname{gr}(V)^{n} \subseteq \operatorname{gr}(E) \operatorname{gr}\left(V^{n}\right) \subseteq \operatorname{gr}\left(E V^{n}\right)
$$

uma vez que

$$
g r\left(E V^{n}\right)=\bigoplus_{i \in \mathbb{Z}} \frac{\left(E V^{n} \cap M_{i}\right)+M_{i-1}}{M_{i-1}} \cong \bigoplus_{i \in \mathbb{Z}} \frac{\left(E V^{n} \cap M_{i}\right)}{\left(E V^{n} \cap M_{i-1}\right)},
$$

e como $\operatorname{dim}_{\mathbb{K}}\left(E V^{n}\right)<\infty$, segue que $\operatorname{dim}_{\mathbb{K}}\left(F W^{n}\right) \leq \operatorname{dim}_{\mathbb{K}}\left(E V^{n}\right)$ para todo inteiro $n>0$. Pela definição da dimensão de Gelfand-Kirillov para módulos, segue o resultado.

Temos interesse especial no caso em que a desigualdade acima é uma igualdade.

Proposição 3.2.8. Seja A uma $\mathbb{K}$-álgebra com filtração finita $\left\{A_{n}\right\}_{i \in \mathbb{Z}}$ tal que gr $(A)$ é finitamente gerado, e seja $M$ um A-módulo à direita com filtração discreta finita $\mathcal{M}=$ $\left\{M_{n}\right\}_{i \in \mathbb{Z}}$ tal que $\operatorname{gr}(M)_{\operatorname{gr}(A)}$ é finitamente gerado. Se $d_{\mathcal{M}}:=\operatorname{dim}_{\mathbb{K}} M_{n}$, para $n \in \mathbb{N}$, então

$$
\mathcal{G}(g r(M))=\mathcal{G}(M)=\mathcal{G}\left(d_{\mathcal{M}}\right)=\mathcal{G}\left(d_{g r(M)}\right),
$$

e portanto, em particular,

$$
\operatorname{GKdim}\left(\operatorname{gr}(M)_{\operatorname{gr}(A)}\right)=G K \operatorname{dim}\left(M_{A}\right)=\overline{\lim } \log _{n} d_{\mathcal{M}}(n)
$$


Demonstração: Por hipótese, existe um número natural $q$ tal que $M_{i}=0$ para todo $i<-q$. Logo

$$
\operatorname{gr}(M)(n)=\bigoplus_{i=-n}^{n} \frac{M_{i}}{M_{i-1}} \cong M_{n}
$$

como $\mathbb{K}$-módulos, para todo $n \geq q$, implicando que $d_{\mathcal{M}} \equiv d_{g r(M)}$, e consequentemente que

$$
\mathcal{G}\left(d_{\mathcal{M}}\right)=\mathcal{G}\left(d_{g r(M)}\right)
$$

Pelo Lema 3.2.3 (b), temos que

$$
\mathcal{G}(\operatorname{gr}(M))=\mathcal{G}\left(d_{\operatorname{gr}(M)}\right) .
$$

Segue das hipóteses que $A$ é uma álgebra finitamente gerada e que $M$ é um $A$-módulo finitamente gerado. Seja $V$ um subespaço gerador de $A$, de dimensão finita e que contém 1, e seja $E$ um subespaço de dimensão finita de $M$ que gera $M$ como $A$-módulo à direita. Existe um número natural $p$ tal que $V \subseteq A_{p}$ e $E \subseteq M_{p}$. Então

$$
E V^{n} \subseteq M_{p} A_{p}^{n} \subseteq M_{p} A_{n p} \subseteq M_{2 p n} \quad \text { para todo } n \geq 1
$$

de forma que

$$
d_{V, E}(n) \leq d_{\mathcal{M}}(2 p n) ; \quad \text { portanto } \mathcal{G}(M) \leq \mathcal{G}\left(d_{\mathcal{M}}\right)
$$

Por fim, temos do lema anterior que

$$
\mathcal{G}(\operatorname{gr}(M))=\mathcal{G}(M)
$$

O resultado acima é amplamente aplicável pois, como o lema seguinte mostra, qualquer módulo finitamente gerado sobre uma $\mathbb{K}$-álgebra filtrada possui uma filtração tal que o módulo graduado associado é finitamente gerado.

Lema 3.2.9. Seja A uma $\mathbb{K}$-álgebra com filtração $\left\{A_{i}\right\}_{i \in \mathbb{Z}}$, e seja $M$ um A-módulo finitamente gerado, $M=E A$, onde E é um subespaço de dimensão finita de $M$. Então $\operatorname{gr}(M)_{\operatorname{gr}(A)}$, o módulo graduado sob a filtração $\left\{E A_{i}\right\}_{i \in \mathbb{Z}}$, é finitamente gerado. 
Demonstração: Seja $x+E A_{i-1} \in E A_{i} / E A_{i-1}$ um elemento homogêneo de $\operatorname{gr}(M)$. Se os elementos $e_{1}, \cdots, e_{m}$ constituem uma base para o espaço vetorial $E \subseteq E A_{0}$, então $x=$ $\sum_{j=1}^{n} e_{j} a_{i j} \operatorname{com} a_{i j} \in A_{i}$. Portanto

$$
x+E A_{i-1}=\sum_{j=1}^{n}\left(e_{j}+E A_{-1}\right)\left(a_{i j}+A_{i-1}\right),
$$

mostrando que os elementos $e_{j}+E A_{-1}, 1 \leq j \leq r$, geram $\operatorname{gr}(M)$ como $g r(A)$-módulo.

Definição 3.2.10. Seja A uma $\mathbb{K}$-álgebra com uma filtração $\left\{A_{i}\right\}_{i \in \mathbb{Z}}$ e seja $M$ um A-módulo finitamente gerado à direita, $M=E A$, para algum subespaço de dimensão finita $E$ de $M$. A filtração $\left\{E A_{i}\right\}_{i \in \mathbb{Z}}$ é uma filtração standard de $M$.

Pela Proposição 3.2.8, o comportamento do crescimento de duas filtrações discretas finitas com módulos graduados associados finitamente gerados é essencialmente o mesmo para qualquer $A$-módulo, onde $A$ é uma $\mathbb{K}$-álgebra finitamente filtrada e $\operatorname{gr}(A)$ é finitamente gerada. De fato, tais filtrações do $A$-módulo à direita $M$ estão ainda mais relacionadas, e são equivalentes no seguinte sentido.

Definição 3.2.11. Sejam $\mathcal{M}=\left\{M_{i}\right\}_{i \in \mathbb{Z}}$ e $\mathcal{N}=\left\{N_{i}\right\}_{i \in \mathbb{Z}}$ duas filtrações do A-módulo à direita $M$, onde $A$ é uma $\mathbb{K}$-álgebra filtrada. Então $\mathcal{M}$ e $\mathcal{N}$ são equivalentes se há um número natural $n$ tal que

$$
N_{i} \subseteq M_{i+n} \quad \text { e } \quad M_{i} \subseteq N_{i+n} \quad \text { para todo } \quad i \in \mathbb{Z}
$$

Proposição 3.2.12. Seja $\mathcal{A}=\left\{A_{i}\right\}_{i \in \mathbb{Z}}$ uma filtração discreta finita de uma $\mathbb{K}$-álgebra $A$, e sejam $\mathcal{M}=\left\{M_{i}\right\}_{i \in \mathbb{Z}}$ e $\mathcal{N}=\left\{N_{i}\right\}_{i \in \mathbb{Z}}$ duas filtrações discretas finitas de um A-módulo à direita $M$. Se $\operatorname{gr}(M)_{\operatorname{gr}(A)}$ é finitamente gerado, então existe um número natural $n$ tal que $M_{i} \subseteq N_{i+n}$ para todo $i \in \mathbb{Z}$.

Demonstração: Como vamos assumir que todas as filtrações são discretas, existe um número natural $q$ tal que

$$
A_{s}=0, \quad e \quad N_{s}=M_{s}=0, \quad \forall s<-q .
$$

Como $\operatorname{gr}(M)_{g r(A)}$ é finitamente gerado, existe um inteiro $r \geq q$ tal que 


$$
g r_{\mathcal{M}}(M)(r)=\bigoplus_{j=-q}^{r} \frac{M_{j}}{M_{j-1}}
$$

é um subespaço gerador de dimensão finita. Como $M_{r}$ possui dimensão finita, existe um número natural $n$ tal que $M_{r} \subseteq N_{n-q}$. Portanto, se $-q \leq i \leq r$, então $n-q \leq n+i$ e consequentemente

$$
M_{i} \subseteq M_{r} \subseteq N_{n-q} \subseteq N_{i+n}
$$

Agora, seja $i>r$, e assuma que $M_{j} \subseteq N_{j+n}$ já foi estabelecido para $j<i$. Como $g r_{\mathcal{M}}(M)(r)$ gera $g r_{\mathcal{M}}(M)$ como $g r(A)$-módulo, segue que

$$
\frac{M_{i}}{M_{i+1}}=\sum_{j=-q}^{r}\left(M_{j} / M_{j-1}\right)\left(A_{i-j} / A_{i-j-1}\right)
$$

e portanto que

$$
M_{i}=\sum_{j=-q}^{r}\left(M_{j} A_{i-j}\right)+M_{i-1} \subseteq \sum_{j=-q}^{r}\left(N_{j+n} A_{i-j}\right)+N_{i-1+n} \subseteq N_{i+n} .
$$

E portanto o resultado segue por indução.

Corolário 3.2.13. Seja A uma $\mathbb{K}$-álgebra discreta e finitamente filtrada, e seja $M$ um Amódulo à direita. Então duas filtrações discretas finitas de $M$ são equivalentes, sempre que seus módulos graduados associados sejam gr $(A)$-módulos à direita finitamente gerados.

Em particular, segue do corolário acima e do Lema 3.2.9 que qualquer filtração discreta e finita de um $A$-módulo finitamente gerado é equivalente à filtração standard.

Lema 3.2.14. Seja $A=\oplus_{i=0}^{\infty} A_{i}$ uma $\mathbb{K}$-álgebra graduada, gerada como álgebra por $A_{1}$, e seja $M=\oplus_{i=0}^{\infty} M_{i}$ um A-módulo graduado à direita com um sistema de geradores homogêneos $\left\{m_{\lambda}\right\}_{\lambda \in \Lambda}$ tal que deg $\left(m_{\lambda}\right) \leq n_{0}$ para algum $n_{0} \in \mathbb{N}$. Então

$$
M_{n+j}=M_{n} A_{j} \quad \text { para todo } \quad n \geq n_{0} \quad \text { e todo } \quad 0 \leq j \in \mathbb{Z}
$$


Demonstração: Seja $n \geq n_{0}$, e seja $m \in M_{n+1}$. Então $m=\sum_{\lambda \in \Lambda_{0}} m_{\lambda} a_{\lambda}$ para um subconjunto finito $\Lambda_{0}$ de $\Lambda$, e podemos supor que os elementos $a_{\lambda} \in A$ são homogêneos de grau $n+1-\operatorname{deg}\left(m_{\lambda}\right)$. Como a álgebra $A$ é gerada por $A_{1}$, e como $\operatorname{deg}\left(A_{\lambda}\right) \geq 0$ para cada $\lambda \in \Lambda_{0}$, cada $a_{\lambda}$ é uma soma de termos da forma $c b$ com $b \in A_{1}$ e um elemento $c \in A$ que é homogêneo de grau $n-\operatorname{deg}\left(m_{\lambda}\right)$. Logo $m \in M_{n} A_{1}$, portanto $M_{n+1}=M_{n} A_{1}$, e o resultado segue por indução.

Como vimos no início deste capítulo, a dimensão de Gelfand-Kirillov nem sempre é exata. Como a exatidão é a chave no estudo de inúmeros resultados relacionados a estrutura de álgebras, o teorema seguinte é importante para o estudo de álgebras noetherianas.

Teorema 3.2.15. Seja $\mathcal{A}=\left\{A_{i}\right\}_{i \in \mathbb{Z}}$ uma filtração discreta finita de uma $\mathbb{K}$-álgebra $A$, tal que a álgebra graduada associada $\operatorname{gr}_{\mathcal{A}}(A)$ é finitamente gerada e noetheriana à direita. Então

$$
\operatorname{GKdim}(M)=\max \{G K \operatorname{dim}(N), G K \operatorname{dim}(P)\}
$$

para toda sequência exata $0 \rightarrow N \rightarrow M \rightarrow P \rightarrow 0$ de A-módulos à direita finitamente gerados.

Demonstração: Como $M_{A}$ é finitamente gerado, $M=E A$ para um subespaço $E$ de dimensão finita. Seja $\mathcal{M}=\left\{E A_{i}\right\}_{i \in \mathbb{Z}}$ a filtração canônica resultante. Pelo Lema 3.2.9, o módulo graduado associado $g r_{\mathcal{M}}(M)$ é finitamente gerado e, portanto, noetheriano, pois $\operatorname{gr}(A)$ é noetheriano à direita por hipótese. As filtrações induzidas

$$
\mathcal{N}=\left\{M_{i} \cap N\right\}_{i \in \mathbb{Z}} \quad \text { e } \mathcal{P}=\left\{\frac{M_{i}+N}{N}\right\}_{i \in \mathbb{Z}}
$$

levam à seqüência exata

$$
0 \rightarrow g r_{\mathcal{N}}(N) \rightarrow g r_{\mathcal{M}}(M) \rightarrow g r_{\mathcal{P}}(P) \rightarrow 0
$$

de $g r(A)$-módulos graduados à direita. Como $g r_{\mathcal{M}}(M)$ é noetheriano, $g r_{\mathcal{N}}(N)$ e $g r_{\mathcal{P}}(P)$ são finitamente gerados. Note que todas as filtrações são finitas e que

$$
d_{\mathcal{M}}(n)=\operatorname{dim}_{\mathbb{K}}\left(M_{n}\right)=\operatorname{dim}_{\mathbb{K}}\left(M_{n} \cap N\right)+\operatorname{dim}_{\mathbb{K}}\left(\left(M_{n}+N\right) / N\right)=d_{\mathcal{N}}(n)+d_{\mathcal{P}}(n)
$$


para qualquer número natural $n$. Segue do Lema 1.2.1 e da Proposição 2.1.2 que

$$
\overline{\lim } \log _{n} d_{\mathcal{M}}(n)=\max \left\{\overline{\lim } \log _{n} d_{\mathcal{N}}(n), \overline{\lim } \log _{n} d_{\mathcal{P}}(n)\right\}
$$

Pela Proposição 3.2.8, temos

$$
\begin{aligned}
\operatorname{GKdim}\left(M_{A}\right) & =\operatorname{GKdim}\left(\operatorname{gr}_{\mathcal{M}}(M)_{\operatorname{gr}(A)}\right)=\overline{\lim } \log _{n} d_{\mathcal{M}}(n) \\
& =\max \left\{\overline{\lim } \log _{n} d_{\mathcal{N}}(n), \overline{\lim } \log _{n} d_{\mathcal{P}}(n)\right\} \\
& =\max \left\{G K \operatorname{dim}\left(g r_{\mathcal{N}}(N)_{\operatorname{gr}(A)}\right), G K \operatorname{dim}\left(g r_{\mathcal{P}}(P)_{\operatorname{gr}(A)}\right)\right\} \\
& =\max \left\{G K \operatorname{dim}\left(N_{A}\right), G K \operatorname{dim}\left(P_{A}\right)\right\} .
\end{aligned}
$$




\section{CAPÍTULO 4}

\section{ÁLGEBRAS}

\section{QUASE-COMUTATIVAS}

\section{1 Álgebras quase-comutativas}

Definição 4.1.1. Uma $\mathbb{K}$-álgebra é quase-comutativa se existe uma filtração

$$
A_{0} \subseteq A_{1} \subseteq \cdots \subseteq \bigcup_{i=0}^{\infty} A_{i}=A
$$

tal que

(a) $A_{0}=\mathbb{K}$

(b) $A_{1}$ tem dimensão finita e $A_{i}=A_{1}^{i}$ para todo $i \geq 1$.

(c) A álgebra graduada associada

$$
\operatorname{gr}(A)=\bigoplus_{i=0}^{\infty} \frac{A_{i}}{A_{i-1}}
$$

é comutativa. 
Pelo Teorema 3.2.15, o resultado seguinte mostra que a dimensão de Gelfand-Kirillov é exata para módulos finitamente gerados sobre uma álgebra quase-comutativa.

Teorema 4.1.2. Seja A uma $\mathbb{K}$-álgebra quase-comutativa com respeito à filtração $\mathcal{A}=$ $\left\{A_{i}\right\}_{i=0}^{\infty}$. Então

(a) $g r_{\mathcal{A}}(A)$ é uma álgebra comutativa noetheriana finitamente gerada.

(b) A e noetheriano à esquerda e à direita.

Demonstração: (b) decorre de (a) pela Proposição 3.2.6. Como uma álgebra, $g r(A)$ é gerada por $A_{1} / A_{0}$, e cada $A_{i} / A_{i-1}$ é gerado pelas palavras de comprimento $i$ compostas por finitos elementos da base o espaço vetorial $A_{1} / A_{0}$. Então existe um homomorfismo de $\mathbb{K}$-álgebras graduadas da álgebra simétrica $S=S\left(A_{1} / A_{0}\right)$ sobre $\operatorname{gr}(A)$. $S$ é noetheriano pelo Teorema da Base de Hilbert, logo $\operatorname{gr}(A)$ é noetheriano.

Exemplo 4.1.3. Seja $A_{n}=A_{n}(\mathbb{K})$ a n-ésima álgebra de Weyl com geradores $x_{1}, \cdots, x_{n}, \partial_{1}, \cdots, \partial_{n}$. Um elemento $a \in A_{n}$ pode ser escrito unicamente como uma combinação linear finita de monômios

$$
x_{1}^{i_{1}} \cdots x_{n}^{i_{n}} \partial_{1}^{j_{1}} \cdots \partial_{n}^{j_{n}}
$$

onde os expoentes são inteiros não-negativos. O grau de tal monômio é a soma de seus expoentes, e o grau deg(a) do elemento a é o maior valor que ocorre como um dos graus dos monômios que compõem a. Isto nos dá uma filtração natural de $A_{n}$ por subespaços de dimensão finita

$$
M_{0}=\mathbb{K}, \quad M_{i}=\left\{a \in A_{n}, \operatorname{deg}(a) \leq i\right\} .
$$

Esta filtração é chamada filtração de Bernstein de $A_{n}$.

Teorema 4.1.4. A álgebra graduada gr $\left(A_{n}\right)$ segundo a filtração de Bernstein é isomorfa ao anel de polinômios sobre $\mathbb{K}$ em $2 n$ variáveis. 
Demonstração: Para $i=1, \cdots, n$, sejam $y_{i}=g r_{1}\left(x_{i}\right)$ e $y_{i+n}=g r_{1}\left(\partial_{i}\right)$. Dividiremos a prova em três partes.

Primeiro passo: $g r\left(A_{n}\right)$ é gerado por $y_{1}, \cdots, y_{2 n}$ como $\mathbb{K}$-álgebra.

É suficiente mostrar este fato para os elementos homogêneos de $\operatorname{gr}\left(A_{n}\right)$. Mas um elemento homogêneo de $g r\left(A_{n}\right)$ é da forma $g r_{k}(d)$ para algum elemento $d$ em $A_{n}$ de grau $k$. Porém $d$ é uma combinação linear de monômios $x^{\alpha} \partial^{\beta}$, com $|\alpha|+|\beta| \leq k$. Se $|\alpha|+|\beta|=k$, então

$$
g r_{k}\left(x^{\alpha} \partial^{\beta}\right)=\left(y_{1}^{\alpha_{1}} \cdots y_{n}^{\alpha_{n}}\right)\left(y_{n+1}^{\beta_{1}} \cdots y_{2 n}^{\beta_{n}}\right) .
$$

Portanto $g r_{k}(d)$ é combinação linear de monômios de grau $k$ em $y_{1}, \cdots, y_{2 n}$, como queríamos demonstrar.

Segundo passo: $\operatorname{gr}\left(A_{n}\right)$ é um anel comutativo.

Como $g r\left(A_{n}\right)$ é gerado por $y_{1}, \cdots, y_{2 n}$, precisamos mostrar apenas que estes elementos comutam em $g r(A)$. Para $i=1, \cdots, n$, temos que $y_{i} y_{i+n}=g r_{2}\left(x_{i} \partial_{i}\right)$ e $y_{i+n} y_{i}=g r_{2}\left(\partial_{i} x_{i}\right)$. Como $\partial_{i} x_{i}=x_{i} \partial_{i}+1$, segue que

$$
g r_{2}\left(\partial_{i} x_{i}\right)=g r_{2}\left(x_{i} \partial_{i}\right)
$$

e portanto $y_{j} y_{i+n}=y_{i+n} y_{j}$. Além disso, para $j \neq i+n$, temos que $y_{i} y_{i+n}=y_{i+n} y_{i}$ uma vez que os elementos correspondentes comutam em $A_{n}$.

Seja $\mathbb{K}\left[z_{i}, \cdots, z_{2 n}\right]$ o anel de polinômios em $2 n$ variáveis. Os dois passos anteriores nos permitem definir um homomorfismo sobrejetor de anéis

$$
\phi: \mathbb{K}\left[z_{i}, \cdots, z_{2 n}\right] \rightarrow \operatorname{gr}\left(A_{n}\right)
$$

por $\phi\left(z_{i}\right)=y_{i}$. Como os $z$ possuem grau 1 em $\mathbb{K}\left[z_{i}, \cdots, z_{2 n}\right]$ e os $y$ possuem grau 1 em $\operatorname{gr}\left(A_{n}\right), \phi$ é um homomorfismo graduado de $\mathbb{K}$-álgebras.

Terceiro passo: $\phi$ é injetor

Seja $F \in \mathbb{K}\left[z_{i}, \cdots, z_{2 n}\right]$, e suponha que $\phi(F)=0$. Como $\phi$ é um homomorfismo graduado, podemos assumir que $F$ é um polinômio homogêneo. Seja

$$
F\left(y_{1}, \cdots, y_{2 n}\right)=\sum c_{\alpha \beta} y_{1}^{\alpha_{1}} \cdots y_{n}^{\alpha_{n}} \cdot y_{n+1}^{\beta_{1}} \cdots y_{2 n}^{\beta_{n}}
$$

onde $\alpha_{1}+\cdots+\alpha_{n}+\beta_{1}+\cdots+\beta_{n}=k$. Defina um operador $d$ de $A_{n}$ pela fórmula 


$$
d=\sum c_{\alpha \beta} x_{1}^{\alpha_{1}} \cdots x_{n}^{\alpha_{n}} \cdot \partial_{1}^{\beta_{1}} \cdots \partial_{n}^{\beta_{n}}
$$

Logo, $\operatorname{gr}_{k}(d)=F\left(y_{1}, \cdots, y_{2 n}\right)$.

Se $g r_{k}(d)=\phi(F)=0$, então $d \in M_{k-1}$. Portanto $d$ pode ser escrito como combinação linear de monômios $x^{\alpha} \partial^{\beta}$ com $|\alpha|+|\beta|<k$. Portanto, pela Proposição 2.3.4, todos os $c_{\alpha \beta}$ acima são iguais a zero. Portanto $F$ é o polinômio nulo e $\phi$ é injetor, como queríamos.

Como já foi mencionado, a dimensão de Gelfand-Kirillov é exata para módulos graduados sobre uma álgebra polinomial comutativa $A$. Como módulos finitamente gerados possuem crescimento polinomial, mostraremos que $G K \operatorname{dim}(M)$ é um inteiro positivo (ou $-\infty$, se $M=0)$. O ponto de partida é um teorema clássico, enunciado por Hilbert, sobre módulos graduados sobre álgebras quase-comutativas. A prova dada é devida a Serre. Os polinômios que surgem são chamados polinômios de Hilbert-Samuel.

Teorema 4.1.5. Seja $A=\mathbb{K}\left[x_{1}, \cdots, x_{r}\right]$ uma álgebra polinomial comutativa, vista como uma álgebra graduada $A=\oplus_{i=0}^{\infty} A_{i}$ via a graduação usual, e seja $M=\oplus_{i=0}^{\infty} M_{i}$ um A-módulo graduado finitamente gerado.

(a) Cada $M_{i}$ é um $\mathbb{K}$-espaço vetorial de dimensão finita.

(b) Para $n$ suficientemente grande, dim $_{\mathbb{K}} M_{n}$ é um polinômio em $n$ de grau $\leq r-1$ com coeficientes racionais.

(c) Para números naturais $n \in \mathbb{N}$ suficientemente grandes,

$$
d_{M}(n):=\operatorname{dim}_{\mathbb{K}}\left(M_{0} \oplus \cdots \oplus M_{n}\right)
$$

é um polinômio em $n$ de grau $\leq r$ com coeficientes racionais.

Demonstração: Para mostrar (a), seja $\left\{m_{1}, \cdots, m_{s}\right\}$ um conjunto de geradores homogêneos de $M$. Para qualquer número natural $q$, um conjunto finito de geradores do $\mathbb{K}$-módulo $M_{q}$ é obtido ao considerar, para cada $j=1, \cdots, s$ todos os elementos $m_{j} \mu_{j l}$, onde $\mu_{j l}$ varia entre todos os monômios em $x_{1}, \cdots, x_{r}$ de grau $q-\operatorname{deg}\left(m_{j}\right)$. 
(b) Vamos proceder por indução sobre $r$. Se $r=0$, então $A=\mathbb{K}$, e como $M$ é finitamente gerado, $M_{n}=0$ para $n$ suficientemente grande. Logo a afirmação vale se fixarmos o grau -1 para o polinômio nulo.

Para $r>0$, defina

$$
\theta: M \rightarrow M \text { por } \theta(m)=x_{r} m
$$

Então $\theta$ é um homomorfismo de grau 1 do $A$-módulo graduado $M$ em si mesmo. Considere a sequência exata

$$
0 \rightarrow K \rightarrow M \stackrel{\theta}{\rightarrow} M \rightarrow C \rightarrow 0
$$

de $A$-módulos graduados, onde $K=\operatorname{Ker}(\theta)$ e $C=\operatorname{Coker}(\theta)$.

Para cada $n$, temos induzida uma sequência exata de $\mathbb{K}$-homomorfismos para as componentes homogêneas

$$
0 \rightarrow K_{n} \rightarrow M_{n} \stackrel{\theta}{\rightarrow} M_{n+1} \rightarrow C_{n+1}
$$

Como $M$ é noetheriano, $C$ e $K$ são finitamente gerados, e são módulos sobre a álgebra

$$
\mathbb{K}\left[x_{1}, \cdots, x_{r-1}\right] \cong \frac{\mathbb{K}\left[x_{1}, \cdots, x_{r}\right]}{\left(x_{r}\right)}
$$

pois $x_{r} C=0=x_{r} K$.

Pela hipótese de indução,

$$
\operatorname{dim}_{\mathbb{K}} K_{n} \quad \text { e } \quad \operatorname{dim}_{\mathbb{K}} C_{n+1}
$$

são polinômios em $n$ de grau menor ou igual a $r-2$, se $n$ é suficientemente grande. Agora

$$
\begin{aligned}
\operatorname{dim}_{\mathbb{K}} C_{n+1} & =\operatorname{dim}_{\mathbb{K}}\left(\frac{M_{n+1}}{\theta\left(M_{n}\right)}\right)=\operatorname{dim}_{\mathbb{K}}\left(M_{n+1}\right)-\operatorname{dim}_{\mathbb{K}}\left(\frac{M_{n}}{K_{n}}\right) \\
& =\operatorname{dim}_{\mathbb{K}}\left(M_{n+1}\right)-\operatorname{dim}_{\mathbb{K}}\left(M_{n}\right)+\operatorname{dim}_{\mathbb{K}}\left(K_{n}\right),
\end{aligned}
$$

pelo Teorema dos Isomorfismos. Logo 


$$
\operatorname{dim}_{\mathbb{K}}\left(M_{n+1}\right)-\operatorname{dim}_{\mathbb{K}}\left(M_{n}\right)=\operatorname{dim}_{\mathbb{K}}\left(C_{n+1}\right)-\operatorname{dim}_{\mathbb{K}}\left(K_{n}\right)
$$

é um polinômio de grau $\leq r-2$ para $n$ suficientemente grande. Portanto $\operatorname{dim}_{\mathbb{K}}\left(M_{n}\right)$ é um polinômio de grau $\leq r-1$ pelo Lema 1.1.6 (b).

(c) Segue do ítem anterior e do Lema 1.1.6 (b).

Corolário 4.1.6. Seja $A=\oplus_{i=0}^{\infty} A_{i}$ uma $\mathbb{K}$-álgebra comutativa tal que $A_{0}=\mathbb{K}$ e $A_{1}$ seja um subespaço gerador de dimensão finita para $A$, e seja $M=\oplus_{i=0}^{\infty} M_{i}$ um A-módulo graduado finitamente gerado. Então, para valores suficientemente grandes de $n$,

$$
d_{M}(n):=\operatorname{dim}_{\mathbb{K}}\left(M_{0} \oplus M_{1} \oplus \cdots \oplus M_{n}\right)
$$

é um polinômio em $n$ com coeficientes racionais e de grau $G K \operatorname{dim}(M)$.

Demonstração: A álgebra $A$ é imagem homomorfa de $S\left(A_{1}\right)$, a álgebra simétrica em $\operatorname{dim}_{\mathbb{K}} A_{1}$ geradores. Logo $M$ é um módulo graduado finitamente gerado sobre uma álgebra polinomial comutativa e a primeira asserção segue do Teorema 4.1.5. Note que como $A \cong \frac{S\left(A_{1}\right)}{I}$, para algum $I \subset S\left(A_{1}\right)$, então $M$ visto como $S\left(A_{1}\right)$-módulo satisfaz $M I=0$. Pela Proposição 3.1 .3 (c), temos que

$$
\operatorname{GKdim}\left(M_{A}\right)=\operatorname{GKdim}\left(M_{S\left(A_{1}\right)}\right)
$$

e pelo Lema 3.2.3 (b) este número é igual ao grau do polinômio $d_{M}$.

Definição 4.1.7. Seja A uma $\mathbb{K}$-álgebra quase-comutativa com respeito à filtração $\mathcal{A}=$ $\left\{A_{i}\right\}_{i \in \mathbb{N}}$, e seja $M$ um A-módulo à direita com filtração $\mathcal{M}=\left\{M_{i}\right\}_{i \in \mathbb{N}}$ tal que o módulo graduado associado $\operatorname{gr}_{\mathcal{M}}(M)$ é finitamente gerado. Para valores suficientemente grandes de $n, a$ função

$$
d_{\mathcal{M}}(n)=\operatorname{dim}_{\mathbb{K}} M_{n}=\operatorname{dim}_{\mathbb{K}}\left(M_{0} \oplus \frac{M_{1}}{M_{0}} \oplus \cdots \oplus \frac{M_{n}}{M_{n+1}}\right)=d_{g r_{\mathcal{M}}(M)}(n)
$$

é um polinômio em n com coeficientes racionais, chamado polinômio de Hilbert-Samuel de $M$ com respeito às filtrações $\mathcal{A}$ e $\mathcal{M}$. Se $d_{\mathcal{M}}(n)$ é escrito como

$$
d_{\mathcal{M}}(n)=a_{d}\left(\begin{array}{l}
n \\
d
\end{array}\right)+a_{d-1}\left(\begin{array}{c}
n \\
d-1
\end{array}\right)+\cdots+a_{1}\left(\begin{array}{l}
n \\
1
\end{array}\right)+a_{0}
$$


então $e(M)=e_{\mathcal{A}, \mathcal{M}}:=a_{d}$ é chamado número de Bernstein de $\boldsymbol{M}$.

Segue da Proposição 3.2.8 que $G K \operatorname{dim}\left(M_{A}\right)$ é o grau de $d_{\mathcal{M}}$. No entanto seu formato particular pode depender de $\mathcal{A}$ e $\mathcal{M}$, mas:

- o grau do polinômio de Hilbert-Samuel de $M$ não depende das filtrações escolhidas para $A$ e $M$,

desde que eles estejam especificados como na definição. Note que o número de Bernstein também pode ser escrito como

(coeficiente dominante de $\left.d_{\mathcal{M}}(n)\right) .(G K \operatorname{dim}(M))$ !

Dadas duas filtrações $\mathcal{M}=\left\{M_{i}\right\}_{i \in \mathbb{N}}$ e $\mathcal{N}=\left\{N_{i}\right\}_{i \in \mathbb{N}}$ de $M$ tais que os módulos $\operatorname{gr}_{\mathcal{M}}(M)$ e $g r_{\mathcal{N}}(N)$ são finitamente gerados (e portanto noetherianos, pois $A$ é noetheriano pela Proposição 4.1.2), então $\mathcal{M}$ e $\mathcal{N}$ são equivalentes pelo Corolário 3.2.13. Logo existe um número natural $q$ tal que

$$
N_{n} \subseteq M_{n+q} \quad \text { e } \quad M_{n} \subseteq N_{n+q} \text { para qualquer } n \in \mathbb{N} \text {. }
$$

Se $e_{\mathcal{M}}(M)$ e $e_{\mathcal{N}}(M)$ são os respectivos números de Bernstein, então para valores suficientemente grandes de $n$ temos que

$$
\begin{aligned}
d_{\mathcal{N}}(n) & =e_{\mathcal{N}}(M)\left(\begin{array}{l}
n \\
d
\end{array}\right)+(\text { termos de grau }<\mathrm{d}) \\
& =\frac{e_{\mathcal{N}}(M)}{d !} n^{d}+(\text { termos de grau }<\mathrm{d}) \\
& \leq d_{\mathcal{M}}(n+q)=e_{\mathcal{M}}(M)\left(\begin{array}{c}
n+q \\
d
\end{array}\right)+(\text { termos de grau }<\mathrm{d}) \\
& =\frac{e_{\mathcal{M}}(M)}{d !} n^{d}+(\text { termos de grau }<\mathrm{d}),
\end{aligned}
$$

e disso segue que $e_{\mathcal{N}}(M) \leq e_{\mathcal{M}}(M)$. Um argumento simétrico nos dá a desigualdade reversa. Portanto $e_{\mathcal{N}}(M)=e_{\mathcal{M}}(M)$. Logo,

- o número de Bernstein de $M$ não depende da filtração particular escolhida para $M$. 
Consequentemente denotaremos o número de Bernstein por $e(M)$, porém, estritamente falando, deve ser feita referência à filtração de $A$, pois o seguinte exemplo mostra que

- o número de Bernstein depende da filtração particular de $A$.

Exemplo 4.1.8. Seja $A=\mathbb{K}[x]$ e sejam $\mathcal{A}=\left\{A_{n}\right\}_{n \in \mathbb{N}}$ e $\mathcal{B}=\left\{B_{n}\right\}_{n \in \mathbb{N}}$ duas filtrações de $A$, dadas por

$$
A_{n}=\mathbb{K}+\mathbb{K} x+\cdots+\mathbb{K} x^{n}, B_{n}=\mathbb{K}+\mathbb{K} x+\cdots+\mathbb{K} x^{2 n}, \text { para } \quad n \geq 1 .
$$

Seja $M=A_{A}$, e tome o subespaço gerador $E=\mathbb{K}$ de $M$. Sejam

$$
\mathcal{M}=\left\{E A_{n}\right\}_{n \in \mathbb{N}} \quad \text { e } \mathcal{N}=\left\{E B_{n}\right\}_{n \in \mathbb{N}}
$$

as filtrações standard resultantes de M. Então

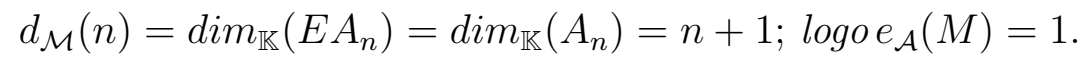

Por outro lado,

$$
d_{\mathcal{N}}(n)=\operatorname{dim}_{\mathbb{K}}\left(E B_{n}\right)=\operatorname{dim}_{\mathbb{K}}\left(B_{n}\right)=2 n+1 ; \operatorname{logoe}_{\mathcal{B}}(M)=2 .
$$

Note que em ambos os casos o número de Bernstein é um inteiro positivo. Pelo Lema 1.1.6 (d), isto sempre ocorre.

Seja $A$ uma álgebra quase-comutativa, e seja

$$
0 \rightarrow L \rightarrow M \stackrel{\phi}{\rightarrow} N \rightarrow 0
$$

uma sequência exata curta de A-módulos à direita finitamente gerados. Sabemos do Teorema 3.2 .15 que

$$
G K \operatorname{dim}(M)=\max \{G K \operatorname{dim}(L), G K \operatorname{dim}(N)\} .
$$

Além disso, se $\mathcal{M}=\left\{M_{n}\right\}_{n \in \mathbb{N}}$ é uma filtração standard de $M$, ou, de fato, é uma filtração para a qual o módulo graduado associado é finitamente gerado, e se $\mathcal{L}=\left(L \cap M_{n}\right)_{n \in \mathbb{N}}$ e $\mathcal{N}=\left(\phi\left(M_{n}\right)\right)_{n \in \mathbb{N}}$ são filtrações induzidas sobre $L$ e $N$, respectivamente, então 


$$
d_{\mathcal{M}}(n)=\operatorname{dim}_{\mathbb{K}}\left(M_{n}\right)=\operatorname{dim}_{\mathbb{K}}\left(M_{n} \cap N\right)+\operatorname{dim}_{\mathbb{K}}\left(\left(M_{n}+N\right) / N\right)=d_{\mathcal{L}}(n)+d_{\mathcal{N}}(n)
$$

para todo $n \in \mathbb{N}$. Como $d_{\mathcal{M}}, d_{\mathcal{L}}$ e $d_{\mathcal{N}}$ são polinômios em $n$ para valores suficientemente grandes de $n$, a exatidão da dimensão de Gelfand-Kirillov para $A$-módulos finitamente gerados segue trivialmente, e a parte (c) do teorema seguinte também vale. Entretanto, a filtração $\mathcal{L}$ induzida sobre $L$ por uma filtração standard $\mathcal{M}$ de $\mathrm{M}$ não é necessariamente uma filtração standard.

Como é vantajoso trabalhar com filtrações standard, a primeira parte do resultado seguinte é importante.

Teorema 4.1.9. Seja A uma $\mathbb{K}$-álgebra quase-comutativa, e seja

$$
0 \rightarrow L \rightarrow M \stackrel{\phi}{\rightarrow} N \rightarrow 0
$$

uma sequência exata curta de A-módulos à direita finitamente gerados. Então

(a) Há filtrações standard $\mathcal{L}, \mathcal{M}$ e $\mathcal{N}$ de $L, M$ e $N$, respectivamente, tais que $d_{\mathcal{M}}(n)=$ $d_{\mathcal{N}}(n)+d_{\mathcal{L}}(n)$ para todo $n \in \mathbb{N}$.

(b) $G K \operatorname{dim}(M)=\max \{G K \operatorname{dim}(L), G K \operatorname{dim}(N)\}$.

(c) Uma das seguintes relações é válida:

$G K \operatorname{dim}(L)<G K \operatorname{dim}(M)=G K \operatorname{dim}(N), e(M)=e(N) ;$

$G K \operatorname{dim}(N)<G K \operatorname{dim}(M)=G K \operatorname{dim}(L), e(M)=e(L)$;

$G K \operatorname{dim}(L)=G K \operatorname{dim}(M)=G K \operatorname{dim}(N), e(M)=e(L)+e(N)$.

Demonstração: Como $d_{\mathcal{L}}, d_{\mathcal{M}}$ e $d_{\mathcal{N}}$ são polinômios em $n$ para valores de $n$ suficientemente grandes, é claro que (b) e (c) seguem de (a). Seja $\mathcal{A}=\left\{A_{n}\right\}_{n \in \mathbb{N}}$ a filtração que torna $A$ quase-comutativa, seja $E$ um subespaço gerador de dimensão finita para $M$, e defina

$$
M_{n}:=E A_{n}, \quad L_{n}:=L \cap M_{n}, \quad \text { e } \quad N_{n}:=\phi\left(M_{n}\right)=\phi(E) A_{n} .
$$

Apesar de $\mathcal{M}=\left\{M_{n}\right\}_{n \in \mathbb{N}}$ e $\mathcal{N}=\left\{N_{n}\right\}_{n \in \mathbb{N}}$ serem filtrações standard de $M$ e $N$, respectivamente, $L=\left\{L_{n}\right\}_{n \in \mathbb{N}}$ não é uma filtração standard de $L$ em geral. Vamos construir 
uma nova filtração standard para $M$ de modo que todas as filtrações induzidas também são standard, e isto conclui o argumento. Notemos que para cada $n$ há uma sequência exata

$$
0 \rightarrow L_{n} \rightarrow M_{n} \rightarrow N_{n} \rightarrow 0
$$

de $\mathbb{K}$-módulos; então obtemos a sequência exata

$$
0 \rightarrow g r_{\mathcal{L}}(L) \rightarrow g r_{\mathcal{M}}(M) \rightarrow g r_{\mathcal{N}}(N) \rightarrow 0
$$

de $\operatorname{gr}(A)$-módulos graduados à direita. Como $\operatorname{gr}(A)$ é um anel noetheriano, e como $g r_{\mathcal{M}}$ é um $\operatorname{gr}(A)$-módulo à direita finitamente gerado, segundo o Lema 3.2.9, $g r_{\mathcal{M}}(M)$ é noetheriano; portanto $g r_{\mathcal{L}}(L)$ é finitamente gerado por, digamos,

$$
L_{0} \oplus \frac{L_{1}}{L_{0}} \oplus \cdots \oplus \frac{L_{m}}{L_{m-1}}
$$

Então

$$
\frac{L_{n}}{L_{n-1}}=\left(\frac{L_{m}}{L_{m-1}}\right) \cdot\left(\frac{A_{n-m}}{A_{n-m-1}}\right) \text { para todo } n \geq m
$$

pelo Lema 3.2.14; portanto

$$
L_{n}=L_{m} A_{n-m}+L_{n-1}
$$

Afirmamos que

$$
L_{n}=L_{m} A_{n-m}, \text { para } n \geq m .
$$

De fato, primeiramente, para $n=m$, temos $L_{m}=L_{m} A_{0}+L_{m-1}$. Mas $L_{m-1}=L_{m-1} .1 \subseteq$ $L_{m} A_{0}$, pois $\mathcal{L}$ é filtração. Agora suponhamos que o resultado vale para $n=k-1 \geq m$ e mostremos que vale para $n=k$. Temos que $L_{k}=L_{m} A_{k-m}+L_{k-1}$. Pela hipótese de indução, $L_{k-1}=L_{m} A_{k-m-1} \subseteq L_{m} A_{k-m}$, de onde segue que $L_{k} \subseteq L_{m} A_{k-m}$, concluindo a afirmação.

Notemos agora que $L_{m}$ é um subespaço gerador de dimensão finita de $L$. Agora escolhemos novas filtrações para $M, N$ e $L$ definindo

$$
M_{0}^{*}=M_{m}, N_{0}^{*}=\phi\left(M_{m}\right)=\phi\left(M_{0}\right), L_{0}^{*}=L_{m},
$$


e, para $n \geq 1$,

$$
\begin{array}{r}
M_{n}^{*}=M_{0}^{*} A_{n}=M_{m+n} \\
N_{n}^{*}=N_{0}^{*} A_{n}=\phi\left(M_{n}^{*}\right) \\
L_{n}^{*}=L_{0}^{*} A_{n}=L_{m} A_{n}=L_{m+n}=L \cap M_{n}^{*} .
\end{array}
$$

Estas são todas filtrações standard, e as filtrações de $L$ e $N$ são induzidas pela nova filtração de $M$.

Corolário 4.1.10. Seja A uma $\mathbb{K}$-álgebra quase-comutativa, e seja $M$ um A-módulo à direita finitamente gerado com $G K \operatorname{dim}(M)=d$ e número de Bernstein e $(M)$. Seja

$$
M=M_{0} \supset M_{1} \supset \cdots \supset M_{i} \supset M_{i+1} \supset \cdots \supset M_{n}
$$

uma cadeia estritamente descendente de submódulos com

$$
\operatorname{GKdim}\left(M_{i} / M_{i+1}\right)=d \quad \text { para } \quad 0 \leq i \leq n-1 .
$$

Então

(a) $e\left(M / M_{i}\right)=\sum_{j=0}^{i-1} e\left(M_{j} / M_{j+1}\right)$.

(b) $n \leq e(M)$.

Demonstração: (a) O resultado será provado por indução sobre $n$. Para $n=1$, não há o que provar. Suponhamos que o resultado tenha sido provado para cadeias de tamanho $k$. Para $n=k+1$, consideremos a seguinte sequência exata:

$$
0 \rightarrow \frac{M_{k}}{M_{k+1}} \rightarrow \frac{M_{0}}{M_{k+1}} \rightarrow \frac{M_{0}}{M_{k}} \rightarrow 0 .
$$

Por hipótese, $G K \operatorname{dim}\left(M_{k} / M_{k+1}\right)=G K \operatorname{dim}\left(M_{0} / M_{1}\right)=d$. Logo, pelo Teorema 4.1.9 (b), segue-se por indução que $G K \operatorname{dim}\left(M_{0} / M_{k}\right)=d$. E portanto, pelo ítem (c) do Teorema 4.1.9,

$$
e\left(M_{0} / M_{k+1}\right)=e\left(M_{0} / M_{k}\right)+e\left(M_{k} / M_{k+1}\right)=\sum_{j=0}^{k} e\left(M_{j} / M_{j+1}\right) .
$$


(b) Pelo Lema 1.1.6 (b), o número de Bernstein de um módulo não nulo é um inteiro positivo, $\log \mathrm{O}$

$$
e\left(M_{j} / M_{j+1}\right) \geq 1 \text { para } 0 \leq j \leq n-1 .
$$

Logo,

$$
n \leq \sum_{j=0}^{n-1} e\left(M_{j} / M_{j+1}\right)=e\left(M / M_{n}\right) \leq e(M)
$$

pela parte (a) e pelo Teorema 4.1 .9 (c).

\subsection{A dimensão de Gelfand-Kirillov de $A_{n}$-módulos}

Como provamos no Teorema 4.1.4, a álgebra graduada associada de $A_{n}$ com respeito à filtração de Bernstein é a álgebra polinomial $\mathbb{K}\left[x_{1}, \cdots, x_{n}, y_{1}, \cdots, y_{n}\right], \operatorname{logo} G K \operatorname{dim}\left(A_{n}\right)=2 n$ (notemos que $A_{n}$ satisfaz as hipóteses da Proposição 3.2.8), como já sabíamos.

Queremos agora mostrar a desigualdade de Bernstein para $A_{n}$, segundo a qual $G K \operatorname{dim}(M) \geq n$ para todo $A_{n}$-módulo $M$ não nulo.

Lema 4.2.1. Sejam $A \subseteq B \mathbb{K}$-álgebras quase-comutativas com respeito às filtrações induzidas pelos subespaços geradores $V \subseteq W$, respectivamente. Seja $M$ um B-módulo finitamente gerado com $G K \operatorname{dim}\left(M_{B}\right)=d$ e número de Bernstein $e_{B}(M)$. Se $N$ é um A-submódulo finitamente gerado de $M$, então

(a) $\operatorname{GKdim}\left(N_{A}\right) \leq d$

(b) $G \operatorname{Kdim}\left(N_{A}\right)=d$ implica que $e_{A}(N) \leq e_{B}(N)$.

Demonstração: (a) Escolha subespaços geradores de dimensão finita $N_{0} \subseteq M_{0}$ para $N_{A}$ e $M_{B}$ respectivamente e defina $N_{n}=N_{0} V^{n}, M_{n}=M_{0} W^{n}$. Então $N_{n} \subseteq M_{n}$ para todo $n$, $\operatorname{logo}$

$$
\begin{aligned}
G K \operatorname{dim}\left(N_{A}\right) & =\overline{\lim } \log _{n} \operatorname{dim}_{\mathbb{K}}\left(N_{n}\right) \\
& \leq \overline{\lim } \log _{n} \operatorname{dim}_{\mathbb{K}}\left(M_{n}\right)=G K \operatorname{dim}\left(M_{B}\right)=d
\end{aligned}
$$


(b) Se $G K \operatorname{dim}\left(N_{A}\right)=G K \operatorname{dim}\left(M_{B}\right)$, então para valores suficientemente grandes de $n$ temos que $\operatorname{dim}_{\mathbb{K}}\left(N_{n}\right)$ e $\operatorname{dim}_{\mathbb{K}}\left(M_{n}\right)$ são polinômios em $n$ de grau $d$ e coeficientes dominantes $e_{A}(n) / d$ ! e $e_{B}(n) / d$ !, respectivamente. Logo, como $\operatorname{dim}_{\mathbb{K}}\left(N_{n}\right) \leq \operatorname{dim}_{\mathbb{K}}\left(M_{n}\right)$, segue que $e_{A}(N) \leq e_{B}(M)$.

O Lema de Quillen é essencial na prova da desigualdade de Bernstein para a dimensão de $A_{n}$-módulos.

Lema 4.2.2. ([6], página 171) Se $M$ é um módulo de comprimento finito sobre uma $\mathbb{K}$ álgebra quase-comutativa $A$, então $\operatorname{End}_{A}(M)$ é algébrico sobre $\mathbb{K}$.

Teorema 4.2.3. Se $M$ é um módulo não nulo à direita sobre a álgebra de Weyl $A_{n}$, então $G \operatorname{Kdim}(M) \geq n$

Demonstração: Por indução em $n$. O caso $n=1$ é o [Corolário 7.13, página 84, [4]], o qual não iremos demonstrar pois foge do objetivo desta dissertação, uma vez que utiliza a dimensão de Krull não comutativa de um módulo $M$. Assuma que para $n \geq 2$ qualquer $A_{n-1}$-módulo possui dimensão de Gelfand-Kirillov pelo menos $n-1$. A álgebra de Weyl $B=A_{n}$ gerada por $x_{1}, \cdots, x_{n}, y_{1}, \cdots, y_{n}$ contém

$$
A=A_{n-1}=\mathbb{K}\left[x_{1}, \cdots, x_{n-1}, y_{1}, \cdots, y_{n-1}\right] \quad \text { e } \quad C=A_{1}=\mathbb{K}\left[x_{n}, y_{n}\right]
$$

como subálgebras. Suponha que $M$ é um $B$-módulo à direita não nulo e finitamente gerado com $G K \operatorname{dim}\left(M_{B}\right)<n$, e seja $N$ um $A$-submódulo não nulo finitamente gerado de $M$. Segue da hipótese de indução que

$$
n-1 \leq G K \operatorname{dim}\left(N_{A}\right) \leq G K \operatorname{dim}\left(M_{A}\right) \leq G K \operatorname{dim}\left(M_{B}\right)<n,
$$

$\log 0$

$$
\operatorname{GKdim}\left(N_{A}\right)=\operatorname{GKdim}\left(M_{B}\right)=n-1 .
$$

Logo, pelo Lema 4.2.1, $e_{A}(N) \leq e_{B}(M)$. Como todo $A$-módulo diferente de 0 possui dimensão de Gelfand-Kirillov pelo menos $n-1$, por indução, o Corolário 4.1.10 mostra que $N$ possui série de composição de comprimento, no máximo, $e_{B}(M)$. Mas $N$ é um $A$ submódulo finitamente gerado arbitrário de $M$. Logo isso implica que $M_{A}$ possui série de 
composição de comprimento no máximo $e_{B}(M)$, e portanto $\operatorname{End}_{A}(M)$ e algébrico sobre $\mathbb{K}$, pelo Lema de Quillen. Como os elementos de $C$ comutam com os elementos de $A$, afirmamos que a álgebra $C$ está mergulhada em $\operatorname{End}_{A}(M)$. De fato, consideremos a aplicação

$$
\Phi: \quad C \rightarrow \operatorname{End}_{A} M \quad c \mapsto \psi_{c}(x)=x c
$$

e mostremos que ela é um mergulho.

(a) $\Phi$ preserva adição

Sejam $c, d \in C$. Então, para todo $x \in M$,

$$
\Phi(c+d)=\psi_{c+d}(x)=x(c+d)=x c+x d=\psi_{c}(x)+\psi_{d}(x)=\Phi(c)+\Phi(d) .
$$

(b) $\Phi$ preserva multiplicação por escalar.

Seja $c \in C, \alpha \in A$. Então, para todo $x \in M$,

$$
\Phi_{c}(x a)=x a c=x c a=\Phi_{c}(x) \cdot a,
$$

uma vez que $c$ comuta com todo elemento de $A$.

(c) $\Phi$ é injetor.

Queremos mostrar que $\operatorname{Ker} \Phi=0$. Seja $c \in \operatorname{Ker} \Phi$. Isto significa que $x c=0$ para todo $x \in M$. Portanto, $c \in r(M)$, o anulador à direita de $M$. Mais ainda, $x \lambda c=0$ para todo $x \in A$, uma vez que $c$ comuta com todos os elementos de $A$. Logo $r(M)$ é um ideal bilateral de $A$. Porém, $A$ é simples e $\Phi$ não é identicamente nula (uma vez que $\Phi(1)$ é a aplicação identidade em $M$. Portanto $r(M)=0$, ou seja, $c=0$ ).

Porém, a existência desse mergulho é uma contradição, uma vez que $C=A_{1}$ não é algébrico sobre $\mathbb{K}$. 


\section{REFERÊNCIAS BIBLIOGRÁFICAS}

[1] ATIYAH, M., MACDONALD, I.C., Introduction to Commutative Algebra, AddisonWesley, London, 1969.

[2] COUTINHO, S.C., A primer of algebraic D-modules, London Mathematical Society Student Texts, vol. 33, Cambridge University Press, Cambridge, 1995.

[3] GOODEARL, K.R., WARFIELD JR., R.B., An introduction to noncommutative noetherian rings, London Mathematical Society Student Texts, Vol. 61, Cambridge University Press, Cambridge, 2004.

[4] KRAUSE, G.R., LENAGAN, T.H., Growth of algebras and Gelfand-Kirillov dimension, Revised Edition, Graduate Studies in Mathematics, vol. 22, American Mathematical Society, 2000.

[5] MILIES, C.P., Anéis e módulos, Publicações do IME-USP, São Paulo, 1973.

[6] QUILLEN, D., On the endomorphism ring of a simple module over an enveloping algebra, Proc. Amer. Math. Soc. 21, 1969, 171-172.

[7] REID, M., Undergraduate commutative algebra, London Mathematical Society Student Texts, Vol. 29, Cambridge University Press, Cambridge, 1995. 